\title{
Bienestar Docente durante la Pandemia de COVID-19 en Chile: Demandas y Recursos para Afrontar la Angustia Psicológica
}

\section{Teacher Well-Being During the COVID-19 Pandemic in Chile: Demands and Resources for Tackling Psychological Distress}

\author{
Verónica Cabezas ${ }^{1,2}$, Marigen Narea ${ }^{3,4}$, David Torres Irribarra ${ }^{3}$, \\ Martín Icaza $^{5}$, Gonzalo Escalona ${ }^{5}$ y Andrés Reyes ${ }^{6}$ \\ ${ }^{1}$ Facultad de Educación, Pontificia Universidad Católica de Chile \\ ${ }^{2}$ Núcleo Milenio en Desarrollo Social, Santiago, Chile \\ ${ }^{3}$ Escuela de Psicología, Pontificia Universidad Católica de Chile \\ ${ }^{4}$ Centro de Estudios Avanzados sobre Justicia Educacional, Santiago, Chile \\ ${ }^{5}$ Elige Educar, Santiago, Chile \\ ${ }^{6}$ Instituto de Economía, Pontificia Universidad Católica de Chile
}

\begin{abstract}
La pandemia por COVID-19 dejó a más de tres cuartas partes de los niños y jóvenes en el mundo sin poder asistir presencialmente a las escuelas durante el año 2020. La crisis está presionando a los educadores y docentes para que cambien su forma de hacer clases, lo que podría estar generando situaciones de mayor estrés para los profesores. Utilizando un cuestionario aplicado online a 6.064 profesores a nivel nacional, por medio de una muestra no probabilística realizada en contexto de pandemia en 2020, se realizaron análisis factoriales exploratorios y confirmatorios y regresiones lineales, usando modelos de ecuaciones estructurales, para analizar el nivel de angustia psicológica de los docentes y su relación con recursos y demandas del entorno del trabajo y factores sociodemográficos y contextuales. Los resultados muestran que principalmente las docentes mujeres, los docentes que están a cargo del cuidado de menores y adultos y los que trabajan en establecimientos particulares pagados son los que se han visto más afectados en su bienestar psicológico. Además, se observa que tener más tiempo disponible para preparar las clases y el poder compatibilizar los tiempos de trabajo y el hogar mejoran el índice de angustia psicológica. Contrario a lo esperado, el contar con más recursos que permita el trabajo a distancia se asocia a un aumento en la angustia psicológica de los docentes. Esto lleva a reflexionar acerca de la necesidad de contar con intervenciones y políticas que se enfoquen en el bienestar docente en el contexto de pandemia que se está viviendo.
\end{abstract}

Palabras clave: COVID-19, angustia psicológica docente, tiempo disponible, modelo recursos-demandas, género

The COVID-19 pandemic left more than three-quarters of the world's children and young people unable to physically attend school. The crisis is putting pressure on educators and teachers to change the way they give lessons, which might be creating more stressful situations for teachers. Based on an online questionnaire administered nationwide to 6,064 Chilean teachers-enrolled through a non-probability sampling strategy during the pandemic in 2020-, exploratory and confirmatory factor analyses and linear regressions were performed, using structural equation modeling, to determine the participants' psychological distress level and how it relates to resources and demands of their educational environment as well as to sociodemographic and contextual factors. Overall, results show that the toll on psychological well-being has been heavier for female teachers, those who take care of minors and adults, and those who work in non-subsidized private schools. In addition, having more time to plan lessons and being able to balance work and home duties was found to reduce teachers' psychological distress index. Contrary to expectations, having more resources to facilitate remote work is associated with an increase in teacher psychological distress. These findings lead to reflection on the need for interventions and policies focused on teacher well-being within the context of the ongoing pandemic.

Keywords: COVID-19, teacher psychological distress, time pressure, resources-demand model, gender

Verónica Cabezas (iD https://orcid.org/0000-0002-3300-8231

Marigen Narea (iD https://orcid.org/0000-0001-7780-7425

Martín Icaza pertenece ahora al Programa Colegios que Aprenden, Enseña Chile, Santiago, Chile.

Este estudio recibió apoyo económico de la Agencia Nacional de Investigación y Desarrollo (ANID) - Programa Iniciativa Científica Milenio - Código NCS17_015/Núcleo Milenio en Desarrollo Social; de ANID - FONDECYT Iniciación (Proyecto N 11180293) y el financiamiento de ANID PIA CIE160007. Los/as autores/as agradecen a Amanda Nogueira de la Pontificia Universidad Católica de Chile y Vanessa Orrego de Elige Educar, por su aporte en el desarrollo de esta investigación, las cuales ayudaron a mejorar sustancialmente la calidad de este estudio. Agradecen también a Elige Educar por facilitarles el acceso al instrumento y la base de datos de la encuesta utilizada en este estudio. No existe ningún conflicto de intereses que revelar además de los mencionados.

La correspondencia relativa a este artículo debe ser dirigida a Verónica Cabezas, Facultad de Educación, Pontificia Universidad Católica de Chile, Avda. Vicuña Mackenna 4860, Macul, Santiago, Región Metropolitana, Chile. Email: vcabezag@uc.cl 
La pandemia de COVID-19 ha llevado a las sociedades en todo el mundo a tomar diversas decisiones para prevenir la propagación del virus y fomentar el cuidado de la población. Como una de las medidas cruciales de prevención, cerca de 178 países cerraron sus establecimientos educacionales durante el 2020 (United Nations Educational, Scientific, and Cultural Organization, 2020). Esta medida ha causado un impacto sin precedentes a la enseñanza tradicional, obligándola a reestructurarse, adoptando nuevas metodologías, con la finalidad de responder a "una enseñanza remota de emergencia" (Carver \& Shanks, 2021; Cheng, 2020; Hodges et al., 2020).

En Chile, alrededor de cinco millones de estudiantes recibieron una educación a distancia en 2020 y cerca de 200.000 docentes enseñaron en forma remota (Ministerio de Educación [MINEDUC], 2020). En este contexto, los profesores están desempeñando un papel clave para garantizar que niños y jóvenes sigan participando y aprendiendo en circunstancias difíciles (Elige Educar, 2020), puesto que la enseñanza se ve limitada por las condiciones existentes (Xie \& Yang, 2020). Por un lado, la crisis está presionando a los educadores para que cambien su forma de hacer clases, usando plataformas, sitios web, enviando guías o usando mensajes de textos (Hinostroza et al., 2020; Yao et al., 2020). Por otro lado, esta pandemia ha puesto en evidencia que siguen existiendo brechas digitales y de acceso entre distintos sectores de la población, que dificultan enormemente el uso de estas tecnologías en el proceso educativo (Agostini \& Willington, 2012; León Aceitón et al., 2019). Estos nuevos desafíos y cambios podrían generar tensión, estrés y angustia en los y las docentes.

A lo anterior se añade que esta pandemia estaría afectando a los docentes al menos desde otras dos dimensiones, desde su propia exposición a la crisis sanitaria y desde su involucramiento con las experiencias de sus alumnos derivadas de esta crisis (Hydon et al., 2015; Pearlman \& Saakvitne, 1995). En cuanto a su propia exposición y la experiencia personal de los docentes, se destaca la problemática de género que emerge en este contexto, ya que dentro del sistema escolar es posible encontrar que un $72,9 \%$ de las docentes son mujeres (Centro de Estudios MINEDUC, 2018). Dado el cierre de escuelas y la extensión de las cuarentenas, la evidencia señala que las mujeres, quienes históricamente han sido las responsable del cuidado informal de la población (Martínez-López et al., 2021), son quienes se han visto más afectadas durante esta crisis (Huarcaya-Victoria, 2020), particularmente aquellas que continúan con su trabajo formal (Martínez-López et al., 2021), dado que resultan perjudicadas con una carga adicional sobre su tiempo por el cuidado de niños, ancianos y enfermos (de Paz et al., 2020; Herrera Ponce et al., 2015; Jofré \& Mendoza, 2005; Rogero García, 2010), sumado al aumento en los niveles ansiedad y estrés que estas sufren, por los riesgo de contagio que conlleva su labor (Van Houtven et al., 2020).

Cabe destacar que históricamente la docencia ha sido calificada como una profesión con altos niveles de estrés (Johnson et al., 2005; Maslach, 1993) debido, entre otras razones, al grado de compromiso emocional de los docentes con las experiencias de sus alumnos (Klassen et al., 2012; López de Lérida Milicic, 2016). Es por esto que, en este ambiente de pandemia, en el que han aumentado los estresores, tanto personales como laborales, es posible pensar que el bienestar psicológico de los profesores se está viendo afectado y, con esto, el aprendizaje de sus estudiantes (Chang, 2009; Gold \& Roth, 1993; Sutton \& Harper, 2009; Yin \& Lee, 2012).

Es por esto que, en el entorno de crisis sanitaria y educativa, este estudio buscó indagar, a través de un modelo de recursos y demanda, qué factores laborales y características sociodemográficas o del contexto se ven asociados a un aumento o disminución de la angustia psicológica de los docentes en Chile durante la pandemia de COVID-19.

\section{Bienestar Docente: Un Foco en la Angustia Psicológica}

Si bien el bienestar docente es de naturaleza multifactorial (De Pablos et al., 2011; Van Horn et al., 2004), en el que se consideran elementos tales como el bienestar afectivo (por ejemplo, satisfacción laboral y angustia psicológica) o conceptualizaciones más amplias del bienestar como, por ejemplo, motivación, en el presente estudio se utiliza el concepto de angustia psicológica como proxy de bienestar docente. Se usa este concepto ya que, en el ámbito docente, la definición de angustia psicológica señala que esta corresponde a una reacción de los docentes a situaciones de hipervigilancia constante, menoscabo de su autoridad, sentimiento de incomprensión y sobrecarga de tareas (Martínez-Otero Pérez, 2003; Zavala Zavala, 2008). Este factor se genera como producto del desequilibrio de las exigencias y presiones que enfrenta el docente en el trabajo y por condiciones laborales no óptimas, las cuales exceden la resistencia y capacidad para resolver problemas (Alvites-Huamaní, 2019). En cierta manera, es lo que se hipotetiza que está ocurriendo hoy con las nuevas exigencias que están enfrentando los docentes en un contexto educacional en constante cambio para adaptarse a la situación de crisis. 
En este sentido, la angustia psicológica actuaría como una respuesta emocional y conductual, que lleva a una disminución del bienestar docente, e influiría en sentimientos de inadecuación, estrés, fracaso, frustración y despersonalización, lo cual le generaría al docente un desajuste en su desempeño profesional (Chiang Vega et al., 2017; Donayre Chang, 2016). También, como mencionan Martinez et al. (2009), la angustia psicológica puede materializarse en sintomatologías físicas, tales como cansancio, pérdida de voz, contracturas corporales, úlceras e hipertensión, siendo estos los padecimientos más comunes.

A pesar de que la angustia psicológica puede ser presentada por cualquier docente (De La Cruz Galarza, 2017), existen características sociodemográficas o de contexto, como el sexo (Barreto Alcoba \& Álvarez, 2013), la edad (Oramas Viera et al., 2007), el tipo de dependencia de las escuelas en que trabajan (Subaldo Suizo, 2012) o el nivel educativo donde se desempeñan (Ávalos \& Sotomayor, 2012; Elige Educar, 2018; Saloviita \& Pakarinen, 2021), que generan heterogeneidad en su aparición o intensidad.

Martínez Ramón (2015) describe que existen diferentes formas de afrontamiento ante las situaciones de estrés según género, siendo las mujeres las que presentan mayores niveles de estrés y burnout, resultados que coinciden con otras investigaciones (León Paime, 2009; Norlund et al., 2010; Zavala Zavala, 2008). En cuanto a la edad, las investigaciones difieren en sus resultados. Si bien existen estudios que no encuentran diferencias entre la edad y el estrés docente (Flores \& Fernández-Castro, 2004), hay estudios que muestran que a medida que transcurren los años, la experiencia podría ser una variable que permita brindar herramientas para afrontar de mejor manera los retos propios de la profesión (González, 2008; Oramas Viera et al., 2007). En relación con el tipo de establecimiento, estudios señalan que en Chile los docentes que se desempeñan en establecimientos privados son menos afectados por el síndrome de burnout que los que trabajan en establecimientos de dependencia municipal (Alvarado et al., 2010; Jorquera Gutiérrez et al., 2014), resultados que difieren de otras investigaciones, como la de Subaldo Suizo (2012), en la que se observa cómo los docentes de establecimientos privados se ven enfrentados a mayores niveles de estrés y ansiedad. Extremera et al. (2010) describen que los problemas de salud más frecuentes vinculados al estrés de los docentes suelen ser diferentes según nivel de impartición. Así, los docentes de primaria presentan una mayor cantidad de síntomas físicos; en cambio, los docentes de secundaria, un mayor nivel de patologías mentales o depresión.

Es por esta heterogeneidad en la asociación entre eventos estresantes y la angustia psicológica presentada por los docentes, es que este estudio tomó en cuenta todas estas características en sus análisis e indagó si estas diferencias también se observaban en la muestra de docentes chilenos estudiados en estado de pandemia de COVID-19.

\section{Bienestar Docente y Modelo de Recursos y Demandas Laborales}

Considerando los antecedentes previamente expuestos y como forma de abordar la angustia psicológica de los docentes durante la pandemia de COVID-19 en Chile, se utilizó el modelo de demanda-recursos laborales (conocido como job demands-resources model, JD-R, en inglés) de Schaufeli y Bakker (2004) para caracterizar el bienestar de estos profesionales. Este modelo explica que las condiciones de empleo pueden categorizarse en demandas y recursos. Estas se relacionan de diferente forma a resultados negativos y positivos, caracterizándose por su flexibilidad y por permitir la inclusión de variables autorreportadas, como lo son la percepción de carga laboral o el tiempo disponible para trabajar. Los recursos laborales se relacionan con los aspectos sociales, psicológicos y organizacionales que permiten sortear o reducir las demandas y costos del trabajo (Lorente Prieto et al., 2008). Estos determinan la manera en que las demandas son percibidas y el estrés que estas generan, ayudando a paliar sus exigencias. Por su lado, las demandas laborales se vinculan con los esfuerzos físicos, psicológicos o sociales que se requieren en la labor profesional, los que están asociados a ciertos costos físicos o psicológicos (Lorente Prieto et al., 2008). A pesar de que estas demandas no necesariamente son negativas, se transforman en estresores laborales cuando requieren un esfuerzo que produce efectos negativos (Schaufeli \& Bakker, 2004).

Las predicciones centrales de este tipo de modelo están representadas por dos procesos duales: (a) el proceso motivacional, en el que los recursos laborales predicen, por ejemplo, el compromiso y (b) el llamado proceso de deterioro de la salud, en el que las demandas laborales predicen la tensión laboral y la angustia psicológica (Bakker \& Demerouti, 2007). Dado que estos procesos se entrelazan a través de efectos directos, indirectos y de interacción, el supuesto central de este modelo es que la tensión laboral surge cuando se han excedido los recursos de una persona (Bakker \& Demerouti, 2007). 
Estudios previos ya han utilizado este modelo para estudiar el bienestar docente (ver Fernet et al., 2013 para una revisión; Hakanen et al., 2006; Llorens et al., 2005; Salanova et al., 2005; Saloviita \& Pakarinen, 2021). Dentro de los recursos laborales considerados, De Neve et al. (2015) establecen tres recursos laborales para analizar su relación con el bienestar docente: la autonomía en el trabajo, el trabajo colaborativo entre pares y el liderazgo transformador de los supervisores directos. En su estudio, encuentran una relación directa entre la autonomía de la tarea y la satisfacción docente, así como con un liderazgo transformacional de sus supervisores directos.

Aun cuando el estudio De Neve et al. (2015) no encuentra una relación entre trabajo colaborativo y bienestar docente, el trabajo colaborativo es uno de los enfoques actuales más importantes para el desarrollo profesional docente (Calvo, 2014). El trabajo colaborativo requiere tanto de herramientas educacionales e instancias de participación, como de la voluntad de las partes involucradas (docentes y directivos) para desarrollar estrategias efectivas de aprendizaje. De esta forma, la promoción y creación de planes de trabajo colaborativo podría resultar efectiva en el combate del estrés y angustia psicológica docente (González-Pérez \& Criado del Pozo, 2006; Guerrero Barona \& Vicente Castro, 2001).

A esos tres recursos laborales se suman los recursos para el trabajo a distancia con que los docentes cuentan, tales como disponer de espacios personales, computador, acceso a la red y celulares para el desarrollo de su práctica profesional. La tecnología como recurso cumple un rol fundamental en los entornos laborales de los docentes, al permitir una comunicación directa y permanente entre los individuos (Ayala Pérez, 2015). Sin embargo, existe escasa evidencia sobre el rol de la tecnología en la profesión docente en contextos de educación remota. Es por esto que se hipotetiza que la tecnología podría verse como una demanda, debido a la sobrecarga que la hiperconectividad puede generar. Para Quan-Haase y Wellman (2006), la hiperconectividad se vincula con la disponibilidad constante de las personas, lo que puede acarrear efectos negativos en los procesos de trabajo, debido a los múltiples requerimientos que se generan en el día a día y a los cuales se deben dar respuesta. También, tal como es destacado por De Carlo et al. (2019), esto genera que los docentes se encuentren cada vez más expuestos a las expectativas y presiones externas (e.g., padres, supervisiones y formuladores de políticas). En el panorama actual, esta difusa línea entre el trabajo y la vida privada de los docentes conduce a sensaciones crónicas de sobrecarga de trabajo, dadas las constantes demandas laborales (Elige Educar, 2020).

En esta investigación, en particular, se exploraron como recursos laborales: la colaboración con los pares, el liderazgo de los supervisores directivos y recursos para el trabajo a distancia, en los que se contempló el uso de tecnologías de información y comunicación, la disponibilidad de un espacio de trabajo, señal de teléfono, un computador personal y conexión a internet. No se incluyó en el análisis la autonomía en el trabajo, ya que las variables de la base de datos utilizada - facilitada por Elige Educar- no permitió considerarla. Elige Educar es una organización sin fines de lucro nacida el año 2009, que funciona de forma autónoma al alero del Centro de Políticas Públicas de la Pontificia Universidad Católica de Chile. Busca impulsar políticas públicas e institucionales que mejoren las condiciones de enseñanza del país.

Entre las demandas laborales, una de las principales identificadas por la literatura es la presión del tiempo disponible (también denominada sobrecarga de trabajo; Klassen \& Chiu, 2010; Kokkinos, 2007; Skaalvik \& Skaalvik, 2011). Skaalvik y Skaalvik (2017) la analizan para predecir el bienestar docente, encontrando que la presión de tiempo predice con un efecto inverso el bienestar de los docentes. Así también, Lorente Prieto et al. (2008), junto a otros autores, analizan la sobrecarga laboral, destacando también el efecto predictor de la sobrecarga laboral en el agotamiento de los docentes (Bakker \& Demerouti, 2007; Kokkinos, 2007; Lorente Prieto et al., 2008; Schaufeli \& Bakker, 2004; Skaalvik \& Skaalvik, 2017). Por su parte, en la investigación realizada por Sarwar et al. (2021) se observa que el tiempo que los docentes destinan a su trabajo por sobre otras áreas — como su vida familiar- resulta importante para determinar la satisfacción que estos poseen con sus trabajos. Entendiendo el contexto de pandemia en el que se realiza esta investigación, resulta necesario reconocer que la presión del tiempo disponible en este periodo de educación a distancia puede verse especialmente afectada por la adecuación de las prácticas pedagógicas, incidiendo en el equilibrio entre las demandas del trabajo y el hogar (De Carlo et al., 2019). De este modo, considerando las variables disponibles para esta investigación, la demanda de la presión del tiempo disponible para el trabajo fue abordada como el tiempo que los profesores poseen para preparar clases y materiales para desarrollar su trabajo desde el hogar y la manera en que estos compatibilizan sus tiempos de trabajo doméstico y trabajo pedagógico. 
En esta misma línea, otra demanda analizada son los retos de la enseñanza remota que han desafiado las metodologías y material didáctico implementados de manera previa por los docentes y la manera en que han tenido que adaptar sus clases, convirtiéndose en otros posibles factores de estrés. El estudio realizado por Xie y Yang (2020) ilustra las adaptaciones que realizó una escuela en la ciudad de Ningbo, China, para cumplir con procesos de enseñanza exitosos durante la pandemia, evidenciando como uno de los retos de la enseñanza remota la modalidad de enseñanza. Debido a la falta de materiales de autoaprendizaje de los estudiantes, el nuevo plan de enseñanza debió contemplar la creación de material didáctico y de videos instructivos o microconferencias y ajustes constantes que permitieran orientar al estudiantado en su desarrollo. Los docentes se encontraron con la necesidad de ajustar el material brindado por las autoridades a los objetivos planteados por el centro educativo, demandando mayor preparación y adaptación (Yao et al., 2020). Para el caso chileno, se han desarrollado capacitaciones a nivel ministerial de uso de plataformas, como Google Classroom (MINEDUC, 2020), y recomendaciones para la gestión curricular, ajustes que han implicado tiempo y capacitaciones por parte de los docentes (Gelber et al., 2021). Así, dentro de la enseñanza remota emerge la modalidad de los procesos educativos como uno de los grandes desafíos en contexto de pandemia. La modalidad en que estos desarrollan su trabajo pedagógico, ya sea a través de clases en vivo, enviando material pedagógico a los estudiantes o realizando grabaciones que permiten una educación asincrónica, o aquellos casos en los que no han realizado clases durante este periodo, puede implicar diferencias en el bienestar laboral que experimentan estos profesionales.

Asimismo, otra demanda que emerge en este estado de cosas es el tamaño de los cursos a los que los docentes deben realizar clases. Se ha señalado que tamaño de cursos más grandes se relacionan con mayores niveles de agotamiento docente y angustia (French, 1993; Travers \& Cooper, 1996). Por otra parte, como se ha mencionado anteriormente, los docentes en esta pandemia deben cumplir el papel de orientador y guía durante los procesos de enseñanza, especialmente en la educación online (Valverde Berrocoso \& Garrido Arroyo, 2005; Yao et al., 2020). Por lo tanto, los grupos sincrónicos, como son los cursos en línea, y los de mayor tamaño disminuyen la participación de todos los integrantes y provoca sobrecarga de información (Wang \& Huang, 2018), lo cual se espera que perjudique el bienestar docente.

Habiendo ya desarrollado los recursos del modelo y descrito las demandas a las que los docentes se enfrentan durante el proceso de enseñanza remota y considerando las variables disponibles en la encuesta que permiten examinar estos factores, es que entre las demandas se exploraron: el tiempo disponible que los docentes poseen para el trabajo, lo cual se mide por el tiempo con el que cuentan para preparar clases y materiales en sus hogares, y la manera como estos declaran haber compatibilizado sus tiempos de trabajo doméstico y trabajo pedagógico. De igual manera, se consideró la modalidad en que los docentes han hecho clases, ya sea en vivo, grabando sus clases o enviando material pedagógico para el aprendizaje del estudiantado, enfrentándose a nuevas demandas laborales, que pueden verse diferenciadas de aquellos docentes que no han realizado clases o enviado material durante este periodo. Como última demanda laboral, se consideró el tamaño del curso, especificado como el número de estudiantes a los que se deben realizar clases.

Finalmente, considerando como los recursos y demandas se relacionan y ayudan a hacer frente de manera efectiva a las demandas laborales y permiten en su conjunto predecir el bienestar laboral (Bakker \& Demerouti, 2013), y la manera en que se desarrollan nuevos desafíos en los contextos de enseñanza remota, la presente investigación buscó describir e interpretar la influencia que ejercen diversos factores predictores en el aumento o disminución de la angustia psicológica.

\section{Bienestar Docente y Ajustes al Sistema Educativo Producto del COVID-19 en Chile}

Sumado a los recursos y demandas laborales anteriormente descritos, para poder realizar un plan de aprendizaje remoto en este contexto, emergen nuevos factores que pueden influir en la angustia psicológica del docente, debido a las condiciones en las que cada uno de estos se encuentra preparando el material, realizando clases y abordando las condiciones que viven sus estudiantes. La literatura reciente revela lo importante que es contar con las condiciones materiales y humanas que permitan desarrollar este tipo de enseñanza de buena manera (Gelber et al., 2021; Pressley, 2021). En Chile, la falta de equidad de acceso a tecnologías y el grado de alfabetización digital de la comunidad educativa (Agostini \& Willington, 2012; León Aceitón et al., 2019) tensionan aún más la realidad escolar de cada docente y los procesos de enseñanza que realizan durante el encierro. Así, en el marco de esta investigación, la ruralidad del establecimiento (Rubio González et al., 2019) o los niveles educativos en los que estos docentes imparten clases (Ávalos \& 
Sotomayor, 2012; Elige Educar, 2018) pueden considerarse predictores del bienestar docente durante la pandemia de Covid-19.

Considerando, además, que una gran mayoría de las docentes son mujeres (Centro de Estudios MINEDUC, 2018), es importante tener en cuenta que, en gran parte de los países, son las mujeres quienes en tiempos normales realizan en mayor medida el trabajo doméstico, crianza de los hijos, orientación, contención y transmisión de conocimientos y cargan con la responsabilidad de cuidar a otras personas (Martínez-Otero, 2003; Toledo González \& Aguilar Pérez, 2016). Por lo tanto, en el estado de pandemia de COVID-19, la carga laboral femenina se ha visto intensificada, debido a que ahora no existen ambientes separados entre el trabajo y la presencia constante de los hijos en el hogar (Andersen et al., 2020). De este modo, es posible pensar que el aumento de presión por las labores domésticas y de cuidado pueden potenciar episodios de angustia psicológica en las docentes mujeres (Martínez-Otero, 2003). Es por esto que, sumado a la evidencia de que existen diferencias en las formas de afrontamiento ante las situaciones de estrés según sexo (Huarcaya-Victoria, 2020; Qiu et al., 2020), en contexto de pandemia de COVID-19 en Chile se hace aún más relevante estudiar las diferencias en la angustia psicológica por género entre los profesionales de la educación, así como estudiar como predictor las labores de cuidado. Esta última no se específica como una demanda en el modelo de demanda-recursos laborales, ya que no corresponde a las condiciones del empleo como tal.

Por lo anterior, este estudio plantea como una primera hipótesis que los recursos y las demandas laborales se relacionan inversa y directamente con el índice de angustia psicológica, respectivamente. Respecto de las características sociodemográficas y de contexto, otra hipótesis que se formula es que el género femenino y poseer labores de cuidado son condiciones que aumentan los niveles de angustia psicológica. Dado que este estudio utilizó la encuesta de Elige Educar "Situación de docente y educadores en contexto de pandemia", que considera una muestra de 6.064 profesores a nivel nacional, permite evaluar estas relaciones mediante modelos estadísticos y un marco sólido de análisis (Bakker \& Demerouti, 2013; Schaufeli \& Bakker, 2004; Xanthopoulou et al., 2007). Además, la encuesta incluye aspectos clave sobre características sociodemográficas (e.g., género, edad), contexto familiar (e.g., cuidado de menores y/o adultos) y ambiente laboral de los docentes (e.g., nivel de enseñanza, ruralidad), lo que permite entender de forma más integral —usando el modelo de demanda y recursos laborales- qué elementos están influyendo en la angustia psicológica de los docentes durante la crisis sanitaria del COVID-19.

\section{Método}

Se desarrolló un estudio observacional de carácter cuantitativo, a través de modelos lineales latentes generalizados (Muthén, 2002; Skrondal \& Rabe-Hesketh, 2004), a partir de una muestra no probabilística de docentes a nivel nacional, contemplando profesionales de diferentes niveles educativos.

\section{Participantes}

La población que contempló la encuesta de Elige Educar es de un total de 190.000 educadores, docentes y directivos del sistema educativo, a los que la organización les hizo llegar el enlace vía correo electrónico para responder un cuestionario. Se obtuvo una muestra no probabilística de 7.187 respuestas en la plataforma. Posteriormente, se excluyeron de la muestra a todos los docentes con funciones directivas que no tenían horas lectivas y tenían exclusividad en su rol directivo o quienes no respondieron si eran o no directivos (en total corresponde a $15,6 \%$ de la base original, equivalente a 1.036 respuestas). Los criterios de exclusión de la muestra de docentes fueron errores en la declaración de variables y casos duplicados. Como resultado de las correcciones, se logró una muestra efectiva de 6.064 observaciones.

En términos de características sociodemográficas, la distribución por género de los 6.064 docentes se compone por 4.807 mujeres (79,3\%), 1.204 hombres (19,9\%) y 53 que no se explicita el género (0,9\%). Respecto a las edades, estas se encontraban comprendidas entre los 19 y 71 años $(M=41,3, D E=10,4)$, observándose en la muestra una edad promedio más alta entre los participantes de género masculino $(M=44,2, D E=11,7)$ que entre las participantes de género femenino $(M=40,6, D E=9,9)$. La muestra incluye a docentes de todas las regiones de Chile, siendo mayoritarias la Metropolitana (39,4\%), Valparaíso (12\%) y Biobío (9,2\%), que son las regiones más pobladas. El $21,9 \%$ de los docentes apoya con el cuidado de algún familiar o persona mayor de edad, mientras que el 12,3\% es el cuidador principal. En la misma línea, el 31,3\% vive con al menos un menor de 7 años dependiente, mientras que el 34,9\% lo hace con menores de edad entre 7 y 17 años. 
Respecto a las características del trabajo, la experiencia laboral promedio de los docentes es de 15,3 años $(D E=9,8)$, observándose en la muestra que es más alta para el género masculino $(M=17,3, D E=11,5)$ que para el femenino $(M=14,9, D E=9,3)$. La mayoría de los docentes trabaja en establecimientos particulares subvencionados $(42,6 \%)$, de un tamaño que no supera los 500 estudiantes (42,7\%) y en zonas urbanas (89,5\%). En relación a su trabajo de aula, la mayoría se encuentra realizando clases en el nivel de enseñanza básica (53,5\%) en cursos con al menos 30 alumnos $(53,5 \%)$. En la Tabla 1 se muestran los estadísticos descriptivos para las variables sociodemográficas y laborales para toda la muestra y por género. Cabe destacar que la muestra, respecto de la población de docentes, se comporta de manera relativamente similar en cuanto al género (la proporción de mujeres es de $79,3 \%$ y $74,5 \%$, respectivamente) y la dependencia (municipales o servicios locales de educación pública son el 36,9\% y 43,6\%; particulares subvencionados, el $43,9 \%$ y $44,6 \%$ y particulares pagados, el $17,4 \%$ y $10,8 \%$, todos respectivamente).

\section{Instrumento}

El instrumento utilizado para realizar el estudio fue un cuestionario online autoaplicado llamado "Situación de docentes y educadores en contexto de pandemia", realizado y aplicado por la organización Elige Educar. El instrumento tiene un total de 113 preguntas, organizadas en tres módulos: (a) condiciones materiales de educadores, docentes y estudiantes; (b) situación personal de educadores y docentes y (c) expectativas del retorno a los establecimientos. Para este estudio se utilizaron preguntas de los dos primeros módulos del cuestionario.

La elaboración del cuestionario por parte de la organización Elige Educar tuvo dos etapas. La primera correspondió a la revisión de instrumentos y literatura relacionada con el levantamiento de información en docentes. La fuente principal fue la metodología de Muñoz Campos et al. (2018), en la que se analiza el bienestar subjetivo y satisfacción vital de los docentes y la influencia que tienen sobre estos factores distintas variables asociadas a elementos sociodemográficos y aspectos relacionados con el desempeño de la labor docente. Adicionalmente, el estudio de Ascorra et al. (2014) aportó un marco conceptual para caracterizar el bienestar social de profesores en contexto de establecimientos educacionales municipales chilenos. Finalmente, informes elaborados por la Organisation for Economic Co-operation and Development (2013; Reimers \& Schleicher, 2020) fueron tomados como referencia para la elaboración de preguntas, para guiar las respuestas de los diversos profesionales de la educación en el contexto de la pandemia de COVID-19. Luego de la revisión de literatura, se realizaron nueve entrevistas a docentes, con el propósito de conocer las condiciones de estos profesionales, sus principales preocupaciones y las dificultades que estaban teniendo, con el fin de ajustar el instrumento a la realidad nacional docente e incorporar nuevas preguntas en función a los hallazgos encontrados.

En una segunda etapa, se procedió a una revisión externa del cuestionario a través de expertos (Blasco Mira et al., 2010), entre los que se consideraron miembros del MINEDUC (División de Educación General, Junta de Jardines Infantiles y Subsecretaría de Educación Parvularia) y 10 docentes con vasta experiencia educativa para los ajustes finales, especialmente adaptación del lenguaje, entre los que se incluyeron profesores de educación parvularia, educación básica y especialistas disciplinarios.

Para este estudio, se consideraron 30 preguntas. En particular, para la elaboración de los índices, se comenzó considerando un subconjunto original de 25 preguntas durante la etapa exploratoria, entre las que fueron finalmente seleccionadas 19 para generar los índices de cinco factores teóricamente relevantes, las que se describen en la sección Análisis de Datos. Estos fueron: la medición de la angustia psicológica (variable dependiente) y los índices asociados a los recursos laborales, recursos para el trabajo a distancia, apoyo directivo y trabajo colaborativo, y el índice asociado a demandas, la demanda de tiempo disponible para el trabajo, la cual se abordó desde la sobrecarga a la que estos profesionales se han tenido que enfrentar en sus trabajos y considera el tiempo que poseen para la preparación de sus clases y la compatibilización del tiempo para el trabajo doméstico y trabajo pedagógico. La selección de los indicadores para estos factores se realizó de forma exploratoria, como se describe en la sección Análisis de Datos. El análisis consideró, además, otras variables asociadas a demandas laborales, tales como el tamaño de curso y los desafíos de la educación remota (preparación de clases y material). A su vez, se incluyeron características sociodemográficas de la muestra, edad, género y experiencia laboral, así como variables contextuales, cuidado principal de menores de edad, apoyo o cuidado principal de mayores de edad, tipo de dependencia del establecimiento educacional y nivel de enseñanza. 
Tabla 1

Estadísticos Descriptivos para las Variables Sociodemográficas Categóricas

\begin{tabular}{|c|c|c|c|}
\hline \multirow{2}{*}{ Variable/categoría } & $\begin{array}{c}\text { Total }^{1} \\
(n=6064)\end{array}$ & $\begin{array}{c}\text { Mujer } \\
(n=4807)\end{array}$ & $\begin{array}{c}\text { Hombre } \\
(n=1204)\end{array}$ \\
\hline & $n(\%)$ & $n(\%)$ & $n(\%)$ \\
\hline \multicolumn{4}{|l|}{ Cuidado de otra persona } \\
\hline Apoyo en cuidado de mayor de edad & $1329(21,9)$ & $1027(21,4)$ & $290(24,1)$ \\
\hline Cuidador principal de mayor de edad & $743(12,3)$ & $637(13,3)$ & $98(8,1)$ \\
\hline Cuidado principal de menor a 7 años & $1899(31,3)$ & $1550(32,2)$ & $337(27,9)$ \\
\hline Cuidado principal de menor entre 7 y 17 años & $2117(34,9)$ & $1747(36,3)$ & $357(29,7)$ \\
\hline \multicolumn{4}{|l|}{ Características del establecimiento donde trabaja } \\
\hline \multicolumn{4}{|l|}{ Tipo de dependencia-1 } \\
\hline Junji & $167(2,8)$ & $162(3,4)$ & $4(0,3)$ \\
\hline Integra & $7(0,1)$ & $7(0,2)$ & $0(0,0)$ \\
\hline Vía transferencia de fondos & $11(0,2)$ & $10(0,2)$ & $1(0,1)$ \\
\hline Municipal & $2168(35,8)$ & $1659(34,5)$ & $484(40,2)$ \\
\hline Particular subvencionado & $2580(42,6)$ & $2065(42,9)$ & $493(40,9)$ \\
\hline Particular Pagado & $1025(16,9)$ & $831(17,3)$ & $190(15,8)$ \\
\hline Administración delegada & $106(1,8)$ & $73(1,5)$ & $32(2,7)$ \\
\hline \multicolumn{4}{|l|}{ Tipo de dependencia-2 (según financiamiento) ${ }^{2}$} \\
\hline Público & $2168(36,5)$ & $1659(35,2)$ & $484(41,3)$ \\
\hline Particular subvencionado & $2580(43,4)$ & $2065(43,8)$ & $493(42,1)$ \\
\hline Particular pagado & $1025(17,3)$ & $831(17,6)$ & $190(16,2)$ \\
\hline \multicolumn{4}{|l|}{ Ubicación } \\
\hline Rural & $638(10,5)$ & $495(10,3)$ & $134(11,1)$ \\
\hline Urbana & $5426(89,5)$ & $4312(89,7)$ & $1070(88,9)$ \\
\hline \multicolumn{4}{|l|}{ Tamaño del establecimiento } \\
\hline Menor de 500 alumnos & $2590(42,7)$ & $2089(43,5)$ & $473(39,3)$ \\
\hline Entre 501 y 1000 alumnos & $1921(31,7)$ & $1517(31,6)$ & $387(32,1)$ \\
\hline Más de 1000 alumnos & $1553(25,6)$ & $1201(24,9)$ & $344(28,6)$ \\
\hline \multicolumn{4}{|l|}{ Nivel educativo de enseñanza } \\
\hline Ed. parvularia (sala cuna y niveles medios) & $317(5,2)$ & $286(5,9)$ & $29(2,4)$ \\
\hline Ed. parvularia (niveles de transición) & $580(9,6)$ & $569(11,8)$ & $7(0,6)$ \\
\hline Ed. básica - primer ciclo & $1690(27,9)$ & $1530(31,8)$ & $144(11,9)$ \\
\hline Ed. básica - segundo ciclo & $1554(25,6)$ & $1167(24,3)$ & $375(31,2)$ \\
\hline Ed. media - científico humanista & $1429(23,6)$ & $956(19,9)$ & $459(38,1)$ \\
\hline Ed. media - técnico profesional & $494(8,1)$ & $299(6,2)$ & $190(15,8)$ \\
\hline \multicolumn{4}{|l|}{ Tamaño del curso } \\
\hline Menor a 15 alumnos & $496(8,2)$ & $426(8,9)$ & $67(5,6)$ \\
\hline Entre 15 y 30 alumnos & $2323(38,3)$ & $1866(38,8)$ & $436(36,2)$ \\
\hline Más de 30 alumnos & $3245(53,5)$ & $2515(52,3)$ & $701(58,2)$ \\
\hline \multicolumn{4}{|l|}{ Modalidad de clases } \\
\hline Realiza clases en vivo & $1102(18,2)$ & $810(16,9)$ & $281(23,3)$ \\
\hline Graba clases / prepara material & $4743(78,2)$ & $3827(79,6)$ & $878(72,9)$ \\
\hline No realiza clases / No prepara material & $219(3,6)$ & $170(3,5)$ & $45(3,8)$ \\
\hline
\end{tabular}




\section{Procedimiento}

El cuestionario fue distribuido electrónicamente de forma individual por la organización Elige Educar, entre el 21 de abril y el 5 de mayo del año 2020, a través de la plataforma de desarrollo de encuestas SurveyMonkey (versión de pago). El tiempo de aplicación fue de 10 a 15 minutos.

$\mathrm{El}$ acceso al cuestionario se realizó mediante un correo electrónico enviado a cada docente con una breve explicación sobre el proceso, el objetivo del estudio y la invitación a participar. Para acceder a este, cada docente debía ingresar al link enviado, el cual incluía un instructivo e información sobre los aspectos éticos.

El proceso de cuidado de información y componente ético se realizó mediante una presentación pública de consentimiento informado en la primera página del cuestionario, el cual debía ser aceptado para iniciar el proceso. En este enunciado se explicitaba el componente de confidencialidad hacia el encuestado, así como el resguardo de la información, la cual no sería compartida fuera de la institución de manera desagregada. Por último, se dejaba de manifiesto la libertad por parte del encuestado de participar de esta instancia, de abandonarla en cualquier momento y la información de contacto del investigador responsable. En este mismo texto se adjuntó un link a otra página que incluía un análisis más detallado del estudio, sus objetivos, la institución y el equipo involucrado.

$\mathrm{El}$ acceso a los registros recolectados a través de la plataforma de encuestas se encontraba limitada al equipo de investigadores.

\section{Análisis de Datos}

El análisis del cuestionario se realizó en dos etapas, en base a un análisis factorial confirmatorio, en un marco de modelos lineales latentes generalizados (Muthén, 2002; Skrondal \& Rabe-Hesketh, 2004). La primera etapa fue exploratoria y se condujo utilizando el $20 \%$ de la muestra (1189 casos); en ella se generaron los modelos de medición de cada uno de los cinco índices que se utilizan en este estudio (variable dependiente, recursos y demandas) y se definió el modelo estructural de las relaciones entre estos índices y las covariables que serían consideradas. La segunda etapa tuvo un carácter confirmatorio, en la cual se ajustaron modelos definidos previamente al $80 \%$ restante de los casos (4875). El procesamiento de datos fue realizado usando R 4.0.0 (R Development Core Team, 2020) y los modelos fueron ajustados utilizando Mplus 8.4 (Muthén \& Muthén, 2017).

\section{Etapa Exploratoria}

Durante la etapa exploratoria se identificaron 19 preguntas del cuestionario que eran informativas de los factores teóricamente relevantes del modelo de recursos y demandas laborales y para examinar la angustia psicológica como medida de bienestar. Dentro de los recursos laborales, recursos para el trabajo a distancia, apoyo directivo, trabajo colaborativo, y dentro de las demandas, tiempo disponible. La decisión respecto a qué preguntas se consideran finalmente como indicadoras de cada uno de los factores fue inicialmente teórica y luego fue revisada con criterios estadísticos, considerando el funcionamiento de los modelos factoriales confirmatorios generalizados de los cinco factores. En base a los análisis realizados en esta etapa, los cinco factores fueron definidos de la siguiente manera (para ver las preguntas exactas utilizadas en cada uno de los índices, consultar el Anexo):

Angustia Psicológica. Este índice incluye siete ítems, incluyendo seis preguntas respecto de la intensidad de sensaciones desde el cierre del establecimiento (ansioso, enojado, relajado (invertido), cansado, preocupado y abrumado) y una pregunta respecto del nivel de estrés ante la nueva modalidad de trabajo. Cada ítem se responde en una escala Likert de 5 puntos $(1=n a d a$ a $5=$ muy para la intensidad sensaciones; $1=$ no me ha afectado a $5=$ me ha afectado bastante en calidad de vida; $1=$ nada estresado a $5=$ muy estresado en nivel de estrés).

Recursos para el Trabajo a Distancia. Para este índice se seleccionaron cuatro preguntas sobre instancias o elementos para desarrollar el trabajo docente desde el hogar, específicamente respecto a la (a) disponibilidad de un espacio de trabajo, (b) señal de teléfono, (c) un computador personal y (d) conexión a Internet en el hogar. Estas preguntas fueron respondidas en una escala Likert de 5 puntos $(1=$ no tengo a $5=$ tengo y es óptimo). 
Apoyo Directivo. Este índice considera, por una parte, preguntas relativas al estado tras el cierre del establecimiento educacional en que trabaja el docente: "me siento apoyado por el equipo directivo en el ámbito pedagógico" y "me siento apoyado por el equipo directivo en el ámbito emocional" y, por otra parte, incluye la evaluación de la orientación técnico-pedagógica desde el establecimiento. Las primeras dos preguntas utilizan una escala de Likert de 5 puntos, donde 1 corresponde a menos que antes y 5 corresponde a más que antes, mientras que en la última pregunta 1 corresponde a no tengo y 5 a tengo y es óptimo.

Trabajo Colaborativo. Incorpora un total de tres ítems, incluyendo preguntas de periodicidad de comunicación con los pares (colegas) por motivos laborales, el grado de satisfacción que sentían en base a dicha comunicación y el cambio en el nivel de trabajo colaborativo, en comparación a antes del cierre del establecimiento. La primera pregunta utiliza como respuesta una escala Likert de 5 puntos, donde 1 corresponde a nunca y 5 corresponde a todos los días, la segunda pregunta utiliza como respuesta una escala Likert de 4 puntos, donde 1 corresponde a muy insatisfecho y 4 corresponde a muy satisfecho, mientras que la última pregunta utiliza un diferencial semántico donde 1 corresponde a menos que antes y 5 a más que antes.

Tiempo Disponible para el Trabajo. En este índice se incluyeron dos ítems: (a) el tiempo para preparar clases y/o material y (b) si los docentes han podido compatibilizar bien los tiempos de trabajo doméstico y trabajo pedagógico. Se utilizó una escala Likert de 5 puntos $(1=$ no tengo a $5=$ tengo y es óptimo para la primera pregunta y $1=$ menos que antes a $5=$ más que antes para la segunda. Con respecto a este índice, es importante destacar que, si bien la presión sobre el tiempo se considera una demanda, los ítems considerados están invertidos. Por lo tanto, producto de la formulación de las preguntas en el cuestionario original, este índice fue formulado de forma inversa, expresando la medida en que el tener un mayor nivel de tiempo disponible se asociaría a más tiempo para el trabajo pedagógico y la preparación de material (y una menor presión sobre el tiempo del trabajo).

\section{Etapa Confirmatoria}

En base a los análisis realizados en la fase exploratoria, se presentan aquí (a) el modelo confirmatorio de medición de angustia psicológica y (b) el modelo estructural que considera todas las variables predictoras y de control relevantes para responder a las preguntas del estudio. El primer modelo evalúa por sí solo el ajuste y confiabilidad del modelo factorial confirmatorio del índice de angustia psicológica. Un segundo modelo expande este modelo, agregando una regresión lineal múltiple, que evalúa el efecto de múltiples covariables (características sociodemográficas y contextuales) sobre angustia psicológica, y además agrega índices de recursos y demandas, en específico recursos para el trabajo a distancia, apoyo directivo, trabajo colaborativo y tiempo disponible, como predictores de la angustia psicológica. También este último modelo agrega otras variables asociadas a las demandas laborales, en particular, tamaño del curso y modalidad de enseñanza en tiempos de educación remota. Estos dos modelos son presentados gráficamente en la Figura 1.

Para evaluar el ajuste de ambos modeles se utilizaron los siguientes índices de ajuste: la raíz del error cuadrático medio de aproximación (RMSEA), el índice comparativo de Bentler-Bonett (CFI) y la raíz del residuo cuadrado medio estandarizado (SRMR) y la prueba $\chi^{2}$. En el caso de RMSEA, los valores menores a 0,05 indican un buen ajuste y mayores a 0,08 indican un mal ajuste. Para el índice CFI, valores superiores a 0,95 indican un buen ajuste del modelo. Los valores de referencia máximos a partir de los cuales se considera que el SRMR indica buen ajuste varían de menos a más exigencia entre < 0,08 y < 0,05 (Hu \& Bentler, 1999; Schermelleh-Engel et al., 2003; Yu, 2002). Finalmente, un buen ajuste en la prueba $\chi^{2}$ es indicado por la ausencia de un resultado estadísticamente significativo.

Como se indicó, los modelos confirmatorios fueron ajustados utilizando Mplus 8.4 (Muthén \& Muthén, 2017), en donde los índices fueron modelados como variables latentes cuantitativas estimadas a través de modelos probit ordinal para dar cuenta del carácter ordinal de las respuestas en escalas Likert. Las escalas de cada índice fueron identificadas, definiendo para cada uno una media de 0 y una desviación estándar de 1. El método de estimación utilizado en todos los casos correspondió a WLSMV (por la sigla en inglés utilizada por el programa Mplus para referirse al estimador de mínimos cuadrados con pesos que incluye medias y varianzas ajustadas).

La precisión de los índices fue estimada utilizando el método de confiabilidad de separación (Andrich, 1982; Verhavert et al., 2018), un método análogo a alfa de Cronbach en el contexto del cálculo de la confiabilidad en modelos de análisis factorial confirmatorio generalizado. 


\section{Figura 1}

Modelos de Medición y Modelos Estructurales Ajustados en la Etapa Confirmatoria

Modelo 1

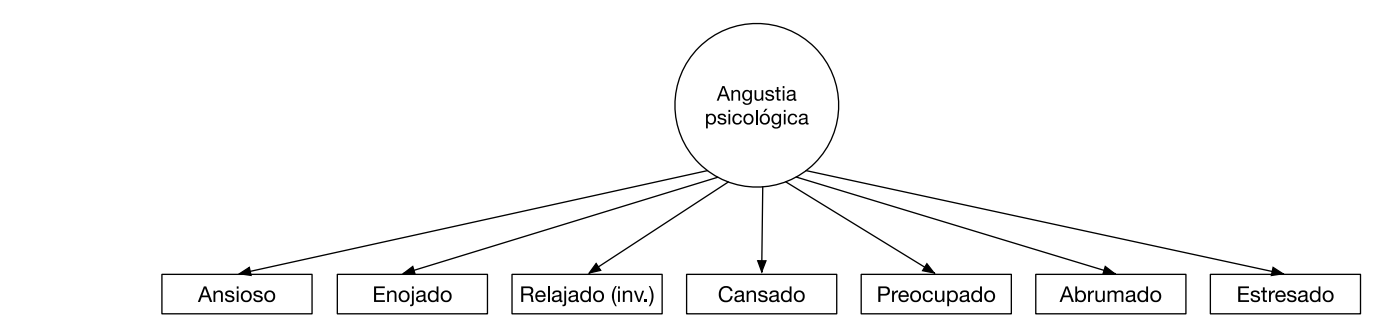

Modelo 2

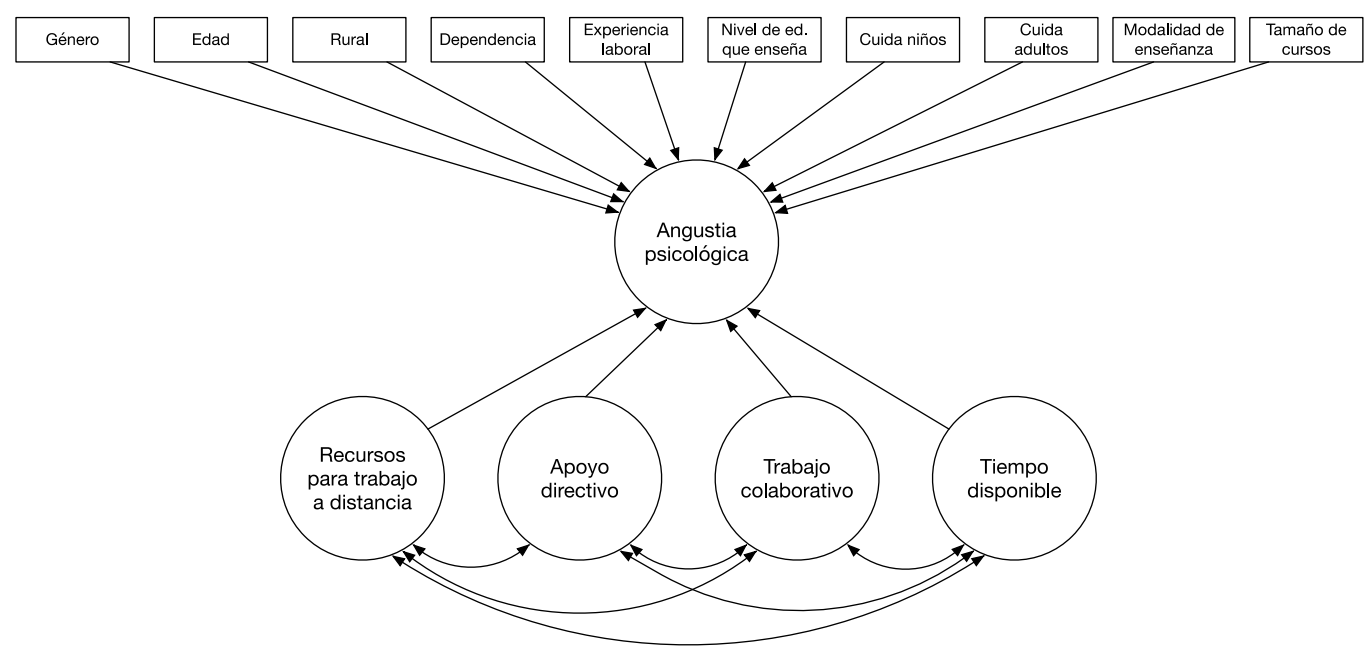

Nota. Cada índice considera un modelo factorial, pero no se muestran para simplificar la presentación.

\section{Resultados}

En base a los modelos definidos en la etapa exploratoria, se realizaron análisis confirmatorios utilizando el $80 \%$ de la muestra que no fue utilizada en la fase exploratoria, evaluando el ajuste y resultados de los modelos. Este análisis fue estructurado en torno a dos modelos sucesivos, comenzando con un primer modelo que evalúa el modelo de medición de la angustia psicológica por sí solo y un segundo modelo que considera simultáneamente la relación de la angustia psicológica con los recursos y demandas considerados en el modelo, controlando los efectos de los predictores sociodemográficos. De esta manera, el primer modelo sirve de base para entender las propiedades métricas de la escala de angustia psicológica, mientras que el segundo modelo contrasta simultáneamente las dos hipótesis del estudio, evaluando en un mismo modelo la hipótesis de (1a) relación directa entre demandas y angustia psicológica, (1b) relación inversa entre recursos y angustia psicológica y (2) la relación directa entre el género femenino y las labores de cuidado con la angustia psicológica.

Los resultados de ajuste para los modelos son presentados en la Tabla 2, la que muestra los índices de ajuste convencionales, a saber, los índices absolutos RMSEA y SRMR, y el índice relativo CFI. En base a estos criterios es posible ver que, a pesar de que hay resultados estadísticamente significativos en todos los casos para la prueba $\chi^{2}$, el ajuste de los modelos varió entre aceptable y bueno en el resto de los índices de ajuste convencionales, en base a lo cual se considera razonable su uso e interpretación en este estudio.

Por su parte, el índice de angustia psicológica tuvo una confiabilidad de separación de 0,83 en el modelo 1 y de 0,85 en el modelo 2 (con pesos factoriales que variaron entre 0,68 y 0,82). Los índices restantes en el modelo 2 tuvieron confiabilidades de separación de 0,79 en el caso de recursos para el trabajo a distancia (con 
pesos factoriales entre 0,64 y 0,83), 0,83 en apoyo directivo (con pesos factoriales entre 0,75 y 0,89), 0,72 en tiempo disponible (con pesos factoriales de 0,77 y -0,71) y 0,57 en colaboración (con pesos factoriales entre $0,29$ y 0,83$)$.

Tabla 2

Índices de Ajuste para los Dos Modelos en la Muestra Confirmatoria

\begin{tabular}{ccccrrc}
\hline Modelo & RMSEA & CFI & SRMR & \multicolumn{1}{c}{$\chi^{2}$} & $g l$ & \multicolumn{1}{c}{$p$} \\
\hline Modelo 1 & 0,057 & 0,992 & 0,017 & 236,065 & 14 & $<0,001$ \\
Modelo 2 & 0,044 & 0,939 & 0,059 & 5100,918 & 484 & $<0,001$ \\
\hline
\end{tabular}

Cabe indicar que, en términos substantivos, los resultados del modelo confirmatorio son consistentes con los que fueron observados en la fase exploratoria. El modelo factorial del índice de angustia psicológica probado en el Modelo 1 fue expandido en el Modelo 2 con regresiones múltiples latentes (De Boeck \& Wilson, 2004) para evaluar los efectos de múltiples predictores. En la Tabla 3 se presentan los valores de los coeficientes de regresión, prediciendo el índice de angustia psicológica por parte de los predictores seleccionados e índices generados. Los resultados presentan al índice de angustia psicológica estandarizado con media 0 y desviación estándar 1, por lo que los coeficientes pueden ser interpretados como el efecto en desviaciones estándar de angustia psicológica de una unidad del predictor.

El Modelo 2 presenta los resultados de la predicción del índice de angustia psicológica una vez incorporadas adicionalmente las demandas y recursos laborales y los factores sociodemográficos y de contexto. Como se puede ver en la Tabla 3, la angustia psicológica es predicha de forma directa por ser mujer, en contraste con hombres, realizar clases en un establecimiento particular pagado, en contraste con quienes hacen clases en colegios municipales, cuidar a un menor de 6 años, en relación a no tener el cuidado de un menor, ser cuidador principal de un adulto o bien apoyar en el cuidado del mismo, en contraste con quienes no reportan tener que cuidar a algún adulto. Por el contrario, la angustia psicológica se asocia inversamente con la edad y con hacer clases en educación parvularia inicial y niveles de transición y directamente con hacer clases en la educación media humanista científico, en relación a hacer clases en educación básica segundo ciclo. Analizando la demanda tamaño del curso, la angustia psicológica es predicha de forma directa por realizar clases en un curso con más de 30 alumnos, en relación a una clase de menos estudiantes. También la demanda de la modalidad de enseñanza en tiempos de educación remota, como realizar clases en vivo, tiene una relación directa, en relación a docentes que declaran no hacer clases y no preparar material. Es posible ver, también, que un aumento en el índice de recursos para el trabajo a distancia y en el de trabajo colaborativo con sus pares se asocian a un aumento de la angustia psicológica. Por otro lado, contar con apoyo directivo no se asoció con la variable de interés, mientras que el tiempo disponible sí lo hizo de forma inversa. Es decir, entre los cuatro índices considerados en el modelo, solo el índice de demanda (que está formulado de forma inversa), contar con más tiempo disponible para el trabajo, predice una disminución de la angustia psicológica. Este último es el predictor de mayor magnitud en el modelo, seguido por el efecto asociado a ser mujer, siendo el primero un factor central que predice un menor nivel de angustia psicológica y el último sería el principal factor que predice un mayor nivel.

Como parte estructural del Modelo 2 se calcularon las correlaciones entres las variables latentes asociadas a los recursos y demandas. En la Tabla 4 se muestran los valores de los coeficientes de correlación entre los índices generados bajo el marco del modelo propuesto. Como se aprecia, existe una correlación directa entre cada factor considerado en el modelo base. Sin embargo, para la finalidad de la predicción de la angustia psicológica, solo tres de los cuatro factores muestran un efecto, siendo apoyo directivo el único índice que no lo hace. De los tres que presentan un efecto, la variable de tiempo disponible muestra el más fuerte y consistente con el modelo de demandas y recursos, ya que esta presentaría el opuesto a una alta presión del tiempo por parte del trabajo. De esta manera, el modelo muestra que un mayor tiempo disponible (i.e., menor demanda de tiempo por parte del trabajo) predice una disminución en la angustia psicológica de los docentes. 
Tabla 3

Modelos Condicionados para Estimar la Relación entre Angustia Psicológica Docente y Variables Predictoras y de Control en la Muestra Confirmatoria

\begin{tabular}{|c|c|}
\hline Angustia psicológica & Modelo 2 \\
\hline Género (Mujer = 1) & $\begin{array}{l}0,423^{* *} \\
(0,038)\end{array}$ \\
\hline Edad & $\begin{array}{l}-0,013^{* *} \\
(0,003)\end{array}$ \\
\hline Ubicación (Urbano = 1) & $\begin{array}{r}0,075 \\
(0,051)\end{array}$ \\
\hline Dependencia particular subvencionado ${ }^{(1)}$ & $\begin{array}{l}-0,059 \\
(0,035)\end{array}$ \\
\hline Dependencia particular pagado ${ }^{(1)}$ & $\begin{array}{l}0,173^{* *} \\
(0,049)\end{array}$ \\
\hline Experiencia laboral & $\begin{array}{r}0,001 \\
(0,003)\end{array}$ \\
\hline Educación parvularia inicial ${ }^{(2)}$ & $\begin{array}{l}-0,188^{*} \\
(0,075)\end{array}$ \\
\hline Educación parvularia transición ${ }^{(2)}$ & $\begin{array}{l}-0,141^{*} \\
(0,057)\end{array}$ \\
\hline Educación básica primer ciclo ${ }^{(2)}$ & $\begin{array}{r}0,078 \\
(0,041)\end{array}$ \\
\hline Educación media humanista científico ${ }^{(2)}$ & $\begin{array}{c}0,090^{*} \\
(0,044)\end{array}$ \\
\hline Educación media técnico profesional ${ }^{(2)}$ & $\begin{array}{r}0,094 \\
(0,062)\end{array}$ \\
\hline Cuida al menos 1 hijo entre 1 y 6 años ${ }^{(3)}$ & $\begin{array}{l}0,232^{* *} \\
(0,034)\end{array}$ \\
\hline Cuida al menos 1 hijo entre 7 y 17 años ${ }^{(3)}$ & $\begin{array}{l}-0,039 \\
(0,032)\end{array}$ \\
\hline Apoya en el cuidado de un adulto ${ }^{(4)}$ & $\begin{array}{l}0,127^{* *} \\
(0,037)\end{array}$ \\
\hline Cuidador principal de un adulto ${ }^{(4)}$ & $\begin{array}{l}0,190^{* *} \\
(0,046)\end{array}$ \\
\hline Realiza clases en vivo ${ }^{(5)}$ & $\begin{array}{c}0,213^{*} \\
(0,086)\end{array}$ \\
\hline Graba vídeos/ prepara material ${ }^{(5)}$ & $\begin{array}{r}0,144 \\
(0,077)\end{array}$ \\
\hline Curso menor de 15 alumnos ${ }^{(6)}$ & $\begin{array}{c}-0,088 \\
(0,058)\end{array}$ \\
\hline Curso con más de 30 alumnos ${ }^{(6)}$ & $\begin{array}{l}0,114^{* *} \\
(0,035)\end{array}$ \\
\hline Recursos para el trabajo a distancia & $\begin{array}{l}0,119^{* *} \\
(0,028)\end{array}$ \\
\hline Apoyo directivo & $\begin{array}{l}-0,036 \\
(0,027)\end{array}$ \\
\hline Trabajo colaborativo & $\begin{array}{l}0,085^{* *} \\
(0,026)\end{array}$ \\
\hline Tiempo disponible & $\begin{array}{l}-0,669^{* *} \\
(0,033)\end{array}$ \\
\hline
\end{tabular}

Nota. $n=4.875$; Desviación estándar entre paréntesis. ${ }^{*} p<0,05 ;{ }^{* *} p<0,001$. Las categorías omitidas en cada variable son las categorías referenciales para las cuales se realiza la estimación. (1) Categoría base: Dependencia municipal; (2) Categoría base: Educación básica segundo ciclo; (3) Categoría base: No cuida a un menor de edad; (4) Categoría base: No se reporta el cuidado de un adulto; (5) Categoría base: No hago clases, no preparo recursos; (6) Categoría base: Curso entre 15 y 30 alumnos. 
Tabla 4

Correlaciones entre los Índices del Modelo 2

\begin{tabular}{lccc}
\hline & $\begin{array}{c}\text { Recursos para el } \\
\text { trabajo a distancia }\end{array}$ & Apoyo directivo & Tiempo disponible \\
\hline Apoyo directivo & 0,406 & 1,000 & 1,000 \\
Tiempo disponible & 0,696 & 0,560 & 0,384 \\
Trabajo colaborativo & 0,273 & 0,640 & \\
\hline
\end{tabular}

Nota. Todos los coeficientes son significativos con $p<0,001$.

\section{Discusión y Conclusiones}

La pandemia de COVID-19 ha provocado un ajuste radical en el sistema educativo, lo que ha provocado cambios y situaciones estresantes para toda la comunidad educativa. En este contexto, los profesores que ya presentaban mayores niveles de estrés en comparación a otras profesiones (Álvarez et al., 1993; Pithers \& Fogarty, 1995), durante esta crisis sanitaria se están viendo enfrentados a un nuevo desafío, como es el trabajo remoto.

En este escenario, este estudio buscó describir cuáles son los predictores de los niveles de angustia psicológica de los docentes y entender si existen ciertas demandas o recursos laborales y personales que podrían afectar negativa o positivamente el nivel de angustia psicológica percibida por los docentes. Para esto se utilizó el modelo de demandas y recursos laborales (Schaufeli \& Bakker, 2004) que fue de ayuda para caracterizar de mejor forma los factores relacionados con la angustia psicológica en una fase exploratoria.

Los resultados muestran que la angustia psicológica se asocia directamente con ser mujer, cuidar a un menor de 6 años, en relación a no tener el cuidado de un menor, y a ser cuidador principal o apoyar en el cuidado de un adulto, en relación a no tener el cuidado de este. Estos resultados van en línea con nuestras hipótesis propuestas y con la literatura expuesta, la cual menciona que este grupo suele verse más afectado en los niveles de estrés y ansiedad que posee, al ser responsable de realizar doble jornada - laboral y de cuidado (de Paz et al., 2020; Herrera Ponce et al., 2015; Jofré \& Mendoza, 2005, Rogero García, 2010)—y por la angustia que genera ser un blanco más cercano de contagio (Van Houtven et al., 2020). Estos resultados están indicando que existen elementos asociados al rol del género femenino y a ser mujer que inciden en los niveles de angustia psicológica de los docentes.

Otros predictores importantes de la angustia psicológica docente se deben al contexto en donde realizan su labor, como es el tipo de establecimiento educacional y el nivel educativo en el que realiza la docencia. Los resultados muestran que los docentes que realizan clases en establecimientos particulares pagados presentan mayor angustia psicológica, en comparación a docentes que hacen clases en un establecimiento municipal o de dependencia de un servicio local de educación. Estos resultados se condicen con lo expuesto por Subaldo Suizo (2012), al indicar que los docentes de centros privados presentan mayores niveles de cansancio emocional y despersonalización con su trabajo. El contexto de pandemia y el rol que cumplen las tecnologías, al permitir una comunicación directa y permanente (Ayala Pérez, 2015), podrían ser una de las razones por las cuales los docentes de estas instituciones presentan una mayor angustia psicológica. La hiperconectividad (Quan-Haase \& Wellman, 2006), junto con la presión y mayores expectativas de aquellos apoderados que son parte de estas comunidades educativas, podrían incidir aún más en este proceso (Quan-Haase \& Wellman, 2006). Por el contrario, hacer clases en el nivel de educación inicial y de transición se asocia a un menor nivel de angustia psicológica, en relación a hacer clases en el nivel del segundo ciclo de educación básica. Este hallazgo va en línea con la investigación realizada por Extremera et al. (2010), que da cuenta de las diferencias en los niveles de estrés y angustia psicológica de los docentes en función del nivel en que estos realizan clases. La literatura brinda luces de que los niveles educativos pueden encontrarse vinculados con los esfuerzos que deben realizar estos profesionales para mantener motivados a sus estudiantes, los niveles de exigencia por parte de los estudiantes según nivel educativo y las diferencias asociadas a la preparación de material para cada grupo etario (Ávalos \& Sotomayor, 2012; Elige Educar, 2018) y como todo esto puede determinar sus niveles de angustia. 
Estos predictores dan cuenta de que existen factores contextuales que predicen el nivel de angustia psicológica de los docentes, lo que va en línea con los estudios existentes que muestran que el bienestar, salud laboral y productividad docente se encuentran fuertemente vinculados con las condiciones laborales donde se desempeñan estos profesionales (Alvites-Huamaní, 2019; Cornejo Chávez, 2009; Johnson et al., 2012).

$\mathrm{Al}$ analizar las demandas y recursos con que cuentan los docentes, se ahondó en cuáles podrían aumentar o disminuir su angustia psicológica y los resultados indican que tanto los recursos para el trabajo a distancia y el de trabajo colaborativo con sus pares se asociaron, si bien con efectos relativamente pequeños en comparación con otros predictores, en forma directa con la angustia psicológica docente. Este último es un resultado que sorprende, ya que va en contra de nuestras hipótesis iniciales y de la evidencia previa que señala al trabajo colaborativo como una estrategia efectiva para evitar el exceso de presión ante el sentimiento de soledad o agobio y que podría resultar efectiva en el combate del estrés y la angustia psicológica docente (González-Pérez \& Criado del Pozo, 2006; Guerrero Barona \& Vicente Castro, 2001). Es posible que las preguntas asociadas al índice desarrollado no den cuenta adecuadamente de elementos relevantes de una colaboración efectiva, la que incluye aspectos que van más allá de la frecuencia y calidad de la comunicación con los pares, como el intercambio de visiones sobre los procesos de enseñanza con foco en los estudiantes. Es importante, sin embargo, que este resultado sea visto con cautela, ya que los coeficientes de precisión/confiabilidad del índice de colaboración creado en este estudio son bajos, lo que debe llevar a tomar con reserva el efecto estimado de este índice en relación a la angustia psicológica docente.

Por su parte, la asociación directa de los recursos disponibles con la angustia psicológica va en la línea con la evidencia previa, que señala que la hiperconectividad (en este caso contar con señal de teléfono, conexión a Internet y un computador en casa) se vincula con la disponibilidad constante de las personas, lo que puede acarrear efectos negativos en los procesos de trabajo, debido a los múltiples requerimientos que se generan en el día a día y a los cuales se debe dar respuesta.

Otro resultado inconsistente con la teoría y nuestras hipótesis es la ausencia de un efecto asociado al apoyo directivo, aun cuando se esperaría que como recurso este se asociara a una disminución en la angustia psicológica docente, en línea con lo propuesto por Thoonen et al. (2011), quienes encuentran una relación directa entre un liderazgo transformacional de sus supervisores directos y la satisfacción docente. La ausencia de una predicción puede deberse a un problema de efectividad en el apoyo directivo percibido o, potencialmente, a que las preguntas que subyacen al índice no logran diferenciar entre un liderazgo transformacional o simplemente brindar los apoyos pedagógicos y emocionales necesarios. Un liderazgo transformacional tiene que ver también con potenciar desarrollo profesional e innovación docente, expresar altas expectativas del desempeño de sus pares y proyectar confianza en el equipo (División de Educación General, 2019).

En relación a las demandas, el efecto del tiempo disponible para el trabajo, entendido como el tiempo que los docentes poseen para preparar clases y materiales para desarrollar su trabajo desde el hogar, y la manera en que compatibilizan sus tiempos de trabajo doméstico y trabajo pedagógico, muestra el efecto más fuerte entre todos los predictores, presentando una relación consistente con estudios previos basados en el modelo de demandas y recursos. Esto va en línea con nuestra hipótesis donde una baja presión sobre el tiempo por parte del trabajo, expresada en una mayor disponibilidad de tiempo y equilibrio vida-trabajo, predeciría una reducción importante en los niveles de angustia psicológica. Considerando que la literatura usualmente se aproxima al tiempo como una demanda que aumenta el malestar en los docentes, es importante considerar que la existencia de tiempo disponible (el opuesto a la presión del tiempo disponible) para que los docentes puedan equilibrar su vida y su trabajo, aparece en este estudio como un factor fundamental al momento de predecir niveles más bajos de angustia psicológica.

Finalmente, se encontró que realizar clases en un curso con más de 30 alumnos aumenta la angustia psicológica, en relación a realizar una clase con menos estudiante. Los cursos más grandes no solo generan mayores niveles de agotamiento docente y angustia (French, 1993; Travers \& Cooper, 1996), sino que también un mayor número de individuos requieren acompañamiento y orientación en este contexto de educación remota (French, 1993; Travers \& Cooper, 1996) y de incertidumbre generalizada constante. En línea con las demandas de una modalidad de enseñanza remota y lo argumentado por Yao et al. (2020) sobre los desafíos de adaptación de clases que deben realizar los docentes en estos contextos, se evidenció que quienes realizan clases en vivo, en contraste con quienes no realizan clases ni preparan materiales, presentan mayores niveles de angustia psicológica. 


\section{Limitaciones}

Este estudio si bien contó con un número importante de docentes encuestados, es importante tener en cuenta que debido a su estrategia de muestreo no tiene representatividad nacional. En segundo lugar, este estudio se basa en un análisis secundario de una base de datos, es por esta razón que no todos los elementos de la teoría de demanda y recursos fueron abordados o no siempre se contó con el mejor indicador para algunos elementos. Aun así creemos que la teoría de demandas y recursos fue un buen marco para analizar nuestros resultados.

\section{Implicancias de Política Pública en Tiempo de COVID-19}

En cuanto a que las mujeres y quienes realizan labores de cuidado presentan mayor angustia psicológica es un resultado relevante, porque en Chile un 72,9\% de las docentes son mujeres (Centro de Estudios MINEDUC, 2018). El rol fundamental que deben cumplir los docentes en situaciones de crisis, como una pandemia (Buckland, 2005; López de Lérida Milicic, 2016; Mutch, 2015; Vernberg et al., 1996), puede verse tensionado en el caso de las docentes cuando este rol coincide con la crianza de los hijos o la responsabilidad de cuidar a otras personas (Martínez-Otero, 2003; Toledo González \& Aguilar Pérez, 2016). Esta labor se ve aún más tensionada cuando la profesora realiza docencia en un establecimiento de dependencia particular, muy probablemente asociado a una mayor hiperconectividad y demandas de diferentes actores (Quan-Haase \& Wellman, 2006). Esto hace fundamental incorporar una perspectiva de género en el abordaje de la emergencia.

En cuanto a los recursos y demandas de los docentes, se encontró que el tiempo es fundamental para cuidar el bienestar docente. En la actual situación de crisis sanitaria, la sobrecarga laboral puede incrementar el horario de trabajo, reduciendo los tiempos de descanso y recreación de los docentes y, por consiguiente, disminuyendo su calidad de vida (Sánchez Cerón \& Corte Cruz, 2012) y perjudicando su balance trabajofamilia (De Carlo et al., 2019).

Se hace necesario, por lo tanto, pensar formas en que los docentes puedan contar con tiempo adecuado para realizar su trabajo en forma remota y disminuir la posible carga en labores administrativas. Una propuesta en relación a este tema es que cada comunidad educativa defina los horarios de trabajo y los límites entre el espacio personal/hogar y el trabajo. En este nuevo contexto las horas lectivas cobran mayor relevancia (Cabezas et al., 2017). Pensar en horarios más reducidos de trabajo sincrónico o dividir tareas entre el equipo docente puede ayudar a contar con más tiempo para la planificación del trabajo. También en esta línea, cobra relevancia la inversión en capacitación docente en herramientas online o procesos que permiten hacer uso más eficiente del tiempo (e.g., enviar mensajes simultáneos, retroalimentaciones grupales), permitiendo disminuir la carga laboral y aumentar el bienestar docente.

Si bien el estudio no encontró asociación entre el trabajo colaborativo entre docentes y el apoyo del equipo directivo, la literatura indica que estos y otros factores organizacionales pueden contribuir a mejorar las condiciones de quienes hacen clases hoy en el país. El hecho de no encontrar apoyo a la relevancia de estos factores puede deberse tanto a la forma de medición como a la calidad del apoyo institucional y la colaboración que se está desarrollando entre docentes. Considerando los resultados de este estudio, pareciera ser que las intervenciones efectivas a corto plazo que busquen potenciar estos factores organizacionales debieran considerar la reducción de carga como un punto de partida, buscando que los docentes dispongan de más tiempo para realizar sus labores pedagógicas y puedan tener un mejor equilibrio trabajo-familia. En esta línea, brindar independencia de las burocracias a los docentes y autonomía en sus trabajos (Hargreaves \& Fullan, 2020), junto con líderes que apoyen y destaquen la labor que los docentes realizan (Weathers, 2011), resultan relevantes en este proceso. Así, también, la literatura destaca como un factor clave la formación de los equipos directivos en este contexto para también garantizar su bienestar y el de sus comunidades (Mutch, 2015; Pascual et al., 2016; Reeves, 2012), junto al trabajo en red entre escuelas que permita el intercambio provechoso de buenas prácticas.

Finalmente, se sabe que al menos en el corto plazo la angustia psicológica de los docentes aumentará a menos que su bienestar sea una prioridad dentro del sistema educativo. Si las escuelas no priorizan el cuidado del bienestar y la salud mental de los docentes, es probable que los niños tengan peores resultados educativos (Roffey, 2012) y se propicien las condiciones para una mayor rotación y deserción docente, aumentando la ya proyectada escasez de profesores idóneos (Medeiros et al., 2018). No se puede esperar que los docentes creen ambientes donde los niños puedan aprender, si ellos sufren de angustia psicológica (Hoglund et al., 2015). 


\section{Futuras direcciones}

Aún cuando hay pocos programas y políticas que se centren en el bienestar de los docentes (Anderson et al., 2019), hay experiencia de programas como "Cultivando la Conciencia y la Resiliencia en la Educación" (CARE, por sus siglas en inglés) que enseña a profesores a manejar el estrés (Jennings et al., 2017) y los métodos de intervención en mindfulness (Taylor et al., 2016). Recientemente, el MINEDUC (2020) lanzó la Bitácora para el Autocuidado Docente) que incluye distintos recursos que promueven la reflexión y el manejo socioemocional. Un camino a seguir podría ser pilotear y evaluar en Chile este tipo de programas ya que podría evitar el deterioro en el bienestar docente (Cinamon et al., 2007; Ilies et al., 2015; Noor \& Zainuddin, 2011) y asegurar un proceso de enseñanza efectivo (Oberle \& Schonert-Reichl, 2016; Roffey, 2012) y, a largo plazo, proteger la salud mental de la comunidad educativa (Glazzard \& Bostwick, 2018; Glazzard \& Rose, 2020).

\section{Referencias}

Agostini, C. A. \& Willington, M. (2012). Acceso y uso de internet en Chile: evolución y factores determinantes. Persona y Sociedad, 26(1), 11-42. https://doi.org/10.11565/pys.v26i1.4

Alvarado, R., Valdivia, L. \& Piñol, D. (2010). Salud mental en los docentes de escuelas municipalizadas y resultados en la prueba SIMCE. Gobierno de Chile, Ministerio de Educación, Fondo de Investigación y Desarrollo en Educación. https://bibliotecadigital.mineduc.cl/bitstream/handle/20.500.12365/18212/E10-0049.pdf?.sequence=1\&isAllowed=y

Álvarez, C., Blanco, J., Aguado, M., Ruiz, A., Cabaco, A., Sánchez, T., Alonso, C. \& Bernabé, J. (1993). Revisión teórica del burnout o desgaste profesional en trabajadores de la docencia. CAESRA, 2(2), 47-65. https://www.researchgate.net/profile/JoseBernabe3/publication/337920753_Revision_teorica_del_burnout_o_desgaste_profesional_en_trabajadores_de_la_docencia_1993_Caesura_2_4765 links/5df38e57a6fdcc28371db378/Revision-teorica-del-burnout-o-desgaste-profesional-en-trabajadores-de-la-docencia 1993-Caesura-2-47-65.pdf

Alvites-Huamaní, C. G. (2019). Estrés docente y factores psicosociales en docentes de Latinoamérica, Norteamérica y Europa. Propósitos $y$ Representaciones, 7(3), 141-159. https://doi.org/10.20511/pyr2019.v7n3.393

Andersen, K. G., Rambaut, A., Lipkin, W. I., Holmes, E. C. \& Garry, R. F. (2020). The proximal origin of SARS-CoV-2. Nature Medicine, 26(4), 450-452. https://doi.org/10.1038/s41591-020-0820-9

Anderson, M., Werner-Seidler, A., King, C., Gayed, A., Harvey, S. B. \& O'Dea, B. (2019). Mental health training programs for secondary school teachers: A systematic review. School Mental Health, 11(3), 489-508. https://doi.org/10.1007/s12310-018-9291-2

Andrich, D. (1982). An index of person separation in latent trait theory, the traditional KR 20 index, and the Guttman Scale response pattern. Education Research and Perspectives, 9(1), 95-104. https://www.rasch.org/erp7.htm

Ascorra, P., López, V., Bilbao, M. A., Correa, T., Guzmán, J., Moraga, V. \& Olavarría, D. (2014). Relación entre el bienestar social de profesores y el nivel de autonomía y tamaño de escuelas municipalizadas chilena. Terapia Psicológica, 32(2), 121-132. https://doi.org/10.4067/S0718-48082014000200005

Ávalos, B. \& Sotomayor, C. (2012). Cómo ven su identidad los docentes chilenos. Perspectiva Educacional: Formación de Profesores, 51(1), 57-86. https://doi.org/10.4151/07189729-Vol.51-Iss.1-Art.74

Ayala Pérez, T. C. (2015). Redes sociales e hiperconectividad en futuros profesores de la generación digital. Ciencia, Docencia y Tecnología, 26(51), 244-270. https://www.redalyc.org/pdf/145/14542676011.pdf

Bakker, A. B. \& Demerouti, E. (2007). The job demands-resources model: State of the art. Journal of Managerial Psychology, 22(3), 309328. https://doi.org/10.1108/02683940710733115

Bakker, A. B. \& Demerouti, E. (2013). La teoría de las demandas y los recursos laborales. Revista de Psicología del Trabajo y de las Organizaciones, 29(3), 107-115. https://doi.org/10.5093/tr2013a16

Barreto Alcoba, C. \& Álvarez, M. E. (2013). Mujeres y docencia. Una mirada desde la historia de vida contada por sus protagonistas. Saber, 25(1), 104-110. http://ve.scielo.org/pdf/saber/v25n1/art12.pdf

Blasco Mira, J. E., López Padrón, A. \& Mengual Andrés, S. (2010). Validación mediante el método Delphi de un cuestionario para conocer las experiencias e interés hacia las actividades acuáticas con especial atención al winsurf. ÁGORA para la Educación Física y el Deporte, 12(1), 75-94. http://dialnet.unirioja.es/servlet/articulo?codigo=3217522

Buckland, P. (2005). Reshaping the future: Education and postconflict reconstruction. World Bank. https://doi.org/10.1596/0-8213-5959-2

Cabezas, V., Medeiros, M. P., Inostroza, D., Gómez, C. \& Loyola, V. (2017). Organización del tiempo docente y su relación con la satisfacción laboral: evidencia para el caso chileno. Archivos Analíticos de Políticas Educativas, 25 , Artículo 64. http://doi.org/10.14507/epaa.25.2451

Calvo, G. (2014). Desarrollo profesional docente: el aprendizaje profesional colaborativo. En Oficina Regional de Educación para América Latina y el Caribe, Temas críticos para formular nuevas políticas docentes en América Latina y el Caribe: el debate actual (pp. 112152). Organización de las Naciones Unidas para la Educación, la Ciencia y la Cultura. https://repositorio.minedu.gob.pe/bitstream/handle/20.500.12799/3775/Temas\%20cr\%C3\%ADticos\%20para\%20formular\%20nuevas\%20pol\%C3 \%ADticas\%20docentes\%20en\%20Am\%C3\%A9rica\%20Latina\%20y\%20el\%20Caribe\%20el\%20debate\%20actual.pdf?sequence=1\&isAllowed=y

Carver, M. \& Shanks, R. (2021). New teachers' responses to COVID-19 in Scotland: Doing surprisingly well? Journal of Education for Teaching, 47(1), 118-120. http://doi.org/10.1080/02607476.2021.1874821

Centro de Estudios Ministerio de Educación. (2018). Análisis base de datos de cargos docentes 2018. Gobierno de Chile, Ministerio de Educación. https://bibliotecadigital.mineduc.cl/bitstream/handle/20.500.12365/14566/minuta_2.pdf?sequence=1\&isAllowed=y

Chang, M L. (2009). An appraisal perspective of teacher burnout: Examining the emotional work of teachers. Educational Psychology Review, 21, 193-218. https://doi.org/10.1007/s10648-009-9106-y

Cheng, X. (2020). Challenges of "school's out, but class's on" to school education: Practical exploration of Chinese schools during the COVID-19 pandemic. Science Insights Education Frontier, 5(2), 501-516. https://.doi.org/10.2139/ssrn.3565605

Chiang Vega, M. M., Heredia Gálvez, S. A. \& Santamaría Freire, E. J. (2017). Clima organizacional y salud psicológica: Una dualidad organizacional. Dimensión Empresarial, 15(1), 63-76. https://doi.org/10.15665/rde.v15i1.641 
Cinamon, R. G, Rich, Y. \& Westman, M. (2007). Teachers' occupation-specific work-family conflict. The Career Development Quarterly, 55(3), 249-261. https://doi.org/10.1002/j.2161-0045.2007.tb00081.x

Cornejo Chávez, R. (2009). Condiciones de trabajo y bienestar/malestar docente en profesores de enseñanza media de Santiago de Chile. Educação \& Sociedade, 30(107), 409-426. https://doi.org/10.1590/S0101-73302009000200006

De Boeck, P. \& Wilson, M. (Eds.). (2004). Explanatory item response models: A generalized linear and nonlinear approach. Springer. https://doi.org/10.1007/978-1-4757-3990-9

De Carlo, A., Girardi, D., Falco, A., Dal Corso, L. \& Di Sipio, A. (2019). When does work interfere with teachers' private life? An application of the job demands-resources model. Frontiers in Psychology, 10, Article 1121. https://doi.org/10.3389/fpsyg.2019.01121

De La Cruz Galarza, E. (2017). Estrés laboral y desempeño docente en la I.E. $N^{\circ}$ 89002, Chimbote, 2017 [Tesis de magíster, Universidad César Vallejo]. $\quad$ Repositorio de la $\quad$ Universidad $\quad$ César https://repositorio.ucv.edu.pe/bitstream/handle/20.500.12692/27735/delacruz_ge.pdf?sequence=1\&isAllowed=y

De Neve, D., Devos, G. \& Tuytens, M. (2015). The importance of job resources and self-efficacy for beginning teachers' professional learning in differentiated instruction. Teaching and Teacher Education, 47(1) 30-41. https://doi.org/10.1016/j.tate.2014.12.003

De Pablos, J. M., Colás Bravo, P. \& González, M. T. (2011). Bienestar docente e innovación con tecnologías de la información y la comunicación. Revista de Investigación Educativa, 29(1), 59-81. https://revistas.um.es/rie/article/view/100131

de Paz, C., Muller, M., Munoz Boudet, A. M. \& Gaddis, I. (2020). Gender dimensions of the COVID-19 pandemic. World Bank. https://doi.org/10.1596/33622

División de Educación General. (2019). Liderazgo escolar: reconociendo los tipos de liderazgo (Guía de Herramientas para el Desarrollo de Recursos Personales en Equipos Directivos: Herramienta 2). Gobierno de Chile, Ministerio de Educación. https://bibliotecadigital.mineduc.cl/bitstream/handle/20.500.12365/14511/HERRAMIENTA2 final.pdf? sequence=1\&isAllowed=y

Donayre Chang, C. E. (2016). Percepción sobre estrés laboral en docentes de educación básica regular de nivel secundario que aplican programas internacionales en una institución educativa privada bilingüe [Tesis de magíster, Pontificia Universidad Católica del Perú]. Repositorio Digital de Tesis y Trabajos de Investigación PUCP. https://tesis.pucp.edu.pe/repositorio/handle/20.500.12404/6945

Elige Educar. (2018). ¿̇Son felices los profesores? Radiografía de la felicidad docente en Chile. https://eligeeducar.cl/content/uploads/2020/07/Estudio-de-felicidad.pdf

Elige Educar. (15 de septiembre de 2020). Situación de docentes y educadores en contexto de pandemia: versión 2 [Diapositivas de PowerPoint]. https://eligeeducar.cl/content/uploads/2020/09/ee-presentacion-covid-2.pdf

Extremera, N., Rey, L. \& Pena, M. (2010). La docencia perjudica seriamente la salud: análisis de los síntomas asociados al estrés docente. Boletín de Psicología, 100, 43-54. https://dialnet.unirioja.es/servlet/articulo?codigo=3391606

Fernet, C., Austin, S., Trépanier, S. -G. \& Dussault, M. (2013). How do job characteristics contribute to burnout? Exploring the distinct mediating roles of perceived autonomy, competence, and relatedness. European Journal of Work \& Organizational Psychology, 22(2), 123-137. https://doi.org/10.1080/1359432X.2011.632161

Flores, M. D. \& Fernández-Castro, J. (2004). Creencias de los profesores y estrés docente en función de la experiencia profesional. Estudios de Psicología, 25(3), 343-357. https://doi.org/10.1174/0210939042450894

French, N. K. (1993). Elementary teacher stress and class size. Journal of Research and Development in Education, 26(2), 66-73. https://psycnet.apa.org/record/1993-35258-001

Gelber, D., Castillo, C., Alarcón, L., Treviño, E. \& Escribano, R. (2021). COVID-19 and the right to education in Chile: An opportunity to revisit our social contract. International Review of Education, 67, 79-101. https://doi.org/10.1007/s11159-021-09881-2

Glazzard, J. \& Bostwick, R. (2018). Positive mental health: A whole school approach. Critical Publishing.

Glazzard, J. \& Rose, A. (2020). The impact of teacher well-being and mental health on pupil progress in primary schools. Journal of Public Mental Health, 19(4), 349-357. https://doi.org/10.1108/JPMH-02-2019-0023

Gold, Y. \& Roth, R. A. (1993). Teachers managing stress and preventing burnout: The professional health solution. Falmer Press.

González, N. (2008). Prevalencia del estrés en la satisfacción laboral de los docentes universitarios. REDHECS: Revista Electrónica de Humanidades, Educación y Comunicación Social, 3(4), 68-89. https://dialnet.unirioja.es/servlet/articulo?codigo=2717959

González-Pérez, J. \& Criado del Pozo, M. J. (2006). Una aproximación a la investigación sobre el estrés laboral en el profesorado. International Journal of Developmental and Educational Psychology, 1(1), 121-129. https://www.redalyc.org/articulo.oa?id=349832311011

Guerrero Barona, E. \& Vicente Castro, F. (2001). Síndrome de "burnout" o desgaste profesional y afrontamiento del estrés en el profesorado. Servicio de Publicaciones de la Universidad de Extremadura.

Hakanen, J. J., Bakker, A. B. \& Schaufeli, W. B. (2006). Burnout and work engagement among teachers. Journal of School Psychology, 43(6), 495-513. https://doi.org/10.1016/j.jsp.2005.11.001

Hargreaves, A. \& Fullan, M. (2020). Professional capital after the pandemic: Revisiting and revising classic understandings of teachers' work. Journal of Professional Capital and Community, 5(3/4), 327-336. https://doi.org/10.1108/JPCC-06-2020-0039

Herrera Ponce, M. S., Fernández Lorca, B., Araya, A. \& Caro, S. (2015). Sistematización y descripción de los perfiles de las cuidadoras de personas dependientes, las demandas de apoyo que las cuidadoras presenten y los programas existentes para aliviar el trabajo de cuidado (incluyendo tareas directas de cuidado, trabajo doméstico, apoyos y acompañamiento de personas dependientes, sean remuneradas o no). Instituto de Sociología de la Pontificia Universidad Católica de Chile.

Hinostroza, J. E., Matamala, C., Ibieta, A., Labbé, C., López, É., Romero, N., González, J., Castillo-Canales, D., Claro, M., Jara, I., Fernández, C. \& Hepp, P. (2020). Docencia durante la crisis sanitaria: la mirada de los docentes. Instituto de Informática Educativa de la Universidad de La Frontera, Observatorio de Prácticas Educativas Digitales de la Facultad de Educación de la Pontificia Universidad Católica de Chile, SUMMA - Laboratorio de Investigación e Innovación en Educación para América Latina y el Caribe,Centro de Desarrollo Profesional Docente de la Facultad de Educación de la Universidad Diego Portales, Centro Costadigital de la Pontificia Universidad Católica de Valparaíso. http://www.miradadocentes.cl/

Hodges, C., Moore, S., Lockee, B., Trust, T. \& Bond, A. (27 de marzo de 2020). The difference between emergency remote teaching and online learning. EDUCAUSE Review. https://er.educause.edu/articles/2020/3/the-difference-between-emergency-remote-teachingand-online-learning

Hoglund, W. L. G., Klingle, K. E. \& Hosan, N. E. (2015). Classroom risks and resources: Teacher burnout, classroom quality and children's adjustment in high needs elementary schools. Journal of School Psychology, 53(5), 337-357. https://doi.org/10.1016/j.jsp.2015.06.002

Hu, L. -t. \& Bentler, P. M. (1999). Cutoff criteria for fit indexes in covariance structure analysis: Conventional criteria versus new alternatives. Structural Equation Modeling, 6(1), 1-55. 
Huarcaya-Victoria, J. (2020). Consideraciones sobre la salud mental en la pandemia de COVID-19. Revista Peruana de Medicina Experimental y Salud Pública, 37(2), 327-334. https://doi.org/10.17843/rpmesp.2020.372.5419

Hydon, S., Wong, M., Langley, A. K., Stein, B. D. \& Kataoka, S. H. (2015). Preventing secondary traumatic stress in educators. Child and Adolescent Psychiatric Clinics of North America, 24(2), 319-333. https://doi.org/10.1016/j.chc.2014.11.003

Ilies, R., Huth, M., Ryan, A. M. \& Dimotakis, N. (2015). Explaining the links between workload, distress, and work-family conflict among school employees: Physical, cognitive, and emotional fatigue. Journal of Educational Psychology, 107(4), 1136-1149. https://doi.org/10.1037/edu0000029

Jennings, P. A., Brown, J. L., Frank, J. L., Doyle, S., Oh, Y., Davis, R., Rasheed, D., DeWeese, A., DeMauro, A. A., Cham, H., \& Greenberg, M. T. (2017). Impacts of the CARE for Teachers program on teachers' social and emotional competence and classroom interactions. Journal of Educational Psychology, 109(7), 1010-1028. https://doi.org/10.1037/edu0000187

Jofré, V. \& Mendoza, S. (2005). Toma de decisiones en salud en mujeres cuidadoras informales. Ciencia y Enfermería, 11(1), 37-49. http://doi.org/10.4067/S0717-95532005000100005

Johnson, S., Cooper, C., Cartwright, S., Donald, I., Taylor, P. \& Millet, C. (2005). The experience of work-related stress across occupations. Journal of Managerial Psychology, 20(2), 178-187. https://doi.org/10.1108/02683940510579803

Johnson, S. M., Kraft, M. A. \& Papay, J. P. (2012). How context matters in high-need schools: The effects of teachers' working conditions on their professional satisfaction and their students' achievement. Teachers College Record, 114(10), 1-39. https://scholar.harvard.edu/files/mkraft/files/johnson_kraft_papay_teacher_working_conditions_final.pdf

Jorquera Gutiérrez, R., Orellana Herrera, C., Tapia Núñez, C. \& Vergara Magnata, E. (2014). Síndrome de burnout en una muestra de profesores/as de enseñanza básica de la ciudad de Copiapó. Summa Psicológica UST, 11(2), 115-134. https://doi.org/10.18774/448x.2014.11.166

Klassen, R. M. \& Chiu, M. M. (2010). Effects of teachers' self-efficacy and job satisfaction: Teacher gender, years of experience, and job stress. Journal of Educational Psychology, 102(3), 741-756. https://doi.org/10.1037/a0019237

Klassen, R. M., Perry, N. E. \& Frenzel, A. C. (2012). Teachers' relatedness with students: An underemphasized component of teachers' basic psychological needs. Journal of Educational Psychology, 104(1), 150-165. https://doi.org/10.1037/a0026253

Kokkinos, C. M. (2007). Job stressors, personality and burnout in primary school teachers. British Journal of Educational Psychology, 77(1), 229-243. https://doi.org/10.1348/000709905X90344

León Aceitón, R., Meza Muñoz, S. \& Araneda Carrasco, T. (2019). Digital readiness Chile. Fundación País Digital \& CISCO USA. https://s3.amazonaws.com/paisdigital/wp-content/uploads/2020/03/30173007/Digital-Readiness-Chile-espa\%C3\%B1ol-online.pdf

León Paime, E. F. (2009). Angustia docente: una revisión de la investigación del malestar y la violencia docente en Latinoamérica. Revista Innovar, Número Extraordinario 1 (Especial en Educación), 91-110. https://www.redalyc.org/pdf/818/81819025008.pdf

Llorens, S., García-Renedo, M. \& Salanova, M. (2005). Burnout como consecuencia de una crisis de eficacia: un estudio longitudinal en profesores de secundaria. Revista de Psicología del Trabajo y las Organizaciones, 21(1-2), 55-70. https://marcalyc.redalyc.org/pdf/2313/231317039004.pdf

López de Lérida Milicic, S. (2016). Tensiones docentes en el año del terremoto y tsunami de 2010 en Chile. Estudios Pedagógicos, 42(3), 259-276. https://doi:10.4067/S0718-07052016000400014

Lorente Prieto, L., Salanova Soria, M., Martínez Martínez, I. \& Schaufeli, W. (2008). Extension of the job demands-resources model in the prediction of burnout and engagement among teachers over time. Psicothema, 20(3), 354-360. https://www.redalyc.org/articulo.oa?id=72720303

Martinez, D., Collazo, M. \& Liss, M. (2009). Dimensiones del trabajo docente: una propuesta de abordage del malestar y el sufrimiento psíquico de los docentes en la Argentina. Educação \& Sociedade, 30(107), 389-408. https://doi.org/10.1590/S0101-73302009000200005

Martínez-López, J. Á., Lázaro-Pérez, C., Gómez Galán, J. \& Raducea, M. (2021). Aproximación a los cuidados de larga duración durante el pico de la COVID-19 en la región de Murcia. Prisma Social, 32, 128-146. https://revistaprismasocial.es/article/view/4073/4783

Martínez-Otero, V. (2003). Autoconcepto docente: análisis de una muestra de profesores y orientadores mexicanos. Revista Educación y Futuro, 14, 25-44. http://files.cer-lacandelaria.webnode.es/200000369-e99a0ea96e/autoconcepto\%20DOCENTE.pdf

Martínez-Otero Pérez, V. (2003). Estrés y ansiedad en los docentes. Pulso: Revista de Educación, 26 , 9-22. https://dialnet.unirioja.es/servlet/articulo? codigo $=499144$

Martínez Ramón, J. P. (2015). Cómo se defiende el profesorado de secundaria del estrés: burnout y estrategias de afrontamiento. Revista de Psicología del Trabajo y de las Organizaciones, 31(1), 1-9. https://doi.org/10.1016/j.rpto.2015.02.001

Maslach, C. (1993). Burnout: A multidimensional perspective. En W. B. Schaufeli, C. Maslach \& T. Marek (Eds.), Professional burnout: Recent developments in theory and research (pp. 19-32). Taylor \& Francis. https://doi.org/10.4324/9781315227979

Medeiros, M. P., Gómez, C., Sánchez, M. J. \& Orrego, V. (2018). Idoneidad disciplinar de los profesores y mercado de horas docentes en Chile. Calidad en la Educación, 48, 50-95. https://doi.org/10.31619/caledu.n48.479

Ministerio de Educación. (14 de mayo de 2020). Apoyos del Mineduc durante la pandemia del Covid-19. Gobierno de Chile. https://www.mineduc.cl/apoyos-del-mineduc-durante-la-pandemia-del-covid-19/

Muñoz Campos, E. M., Fernández González, A. \& Jacott, L. (2018). Bienestar subjetivo y satisfacción vital del profesorado. REICE. Revista Iberoamericana sobre Calidad, Eficacia y Cambio en Educación, 16(1), 105-117. https://doi.org/10.15366/reice2018.16.1.007

Mutch, C. (2015). The role of schools in disaster settings: Learning from the 2010-2011 New Zealand earthquakes. International Journal of Educational Development, 41, 283-291. https://doi.org/10.1016/j.ijedudev.2014.06.008

Muthén, B. O. (2002). Beyond SEM: General latent variable modeling. Behaviormetrika, 29(1), 81-117. https://doi.org/10.2333/bhmk.29.81

Muthén, L. K. \& Muthén, B. O. (2017). Mplus: Statistical analysis with latent variables, user's guide (8a ed.) [Software computacional]. https://www.statmodel.com/html_ug.shtml

Noor, N. M. \& Zainuddin, M. (2011). Emotional labor and burnout among female teachers: Work-family conflict as mediator. Asian Journal of Social Psychology, 14(4), 283-293. https://doi.org/10.1111/j.1467-839X.2011.01349.x

Norlund, S., Reuterwall, C., Höög, J., Lindahl, B., Janlert, U. \& Birgander, L. S. (2010). Burnout working conditions and gender - results from the northern Sweden MONICA Study. BMC Public Health, 10, Artículo 326. https://doi.org/10.1186/1471-2458-10-326

Oberle, E. \& Schonert-Reichl, K. A. (2016). Stress contagion in the classroom? The link between classroom teacher burnout and morning cortisol in elementary school students. Social Science \& Medicine, 159, 30-37. https://doi.org/10.1016/j.socscimed.2016.04.031

Oramas Viera, A., Almirall Hernandez, P. \& Fernández, I. (2007). Estrés laboral y el síndrome de burnout en docentes venezolanos. Salud de los Trabajadores, 15(2), 71-87. https://www.redalyc.org/pdf/3758/375839287002.pdf

Organisation for Economic Co-operation and Development. (2013). OECD guidelines on measuring subjective well-being. https://doi.org/10.1787/9789264191655-en 
Pascual, J., Larraguibel, D., Zenteno, D. \& Guarda, F. (2016). Liderazgo escolar en tiempos de crisis. El caso de dos liceos del centro sur de Chile después del 27F. REICE. Revista Iberoamericana sobre Calidad, Eficacia y Cambio en Educación, 14(2), 45-62. https://doi.org/10.15366/reice2016.14.2.003

Pearlman, L. A. \& Saakvitne, K. W. (1995). Treating therapists with vicarious traumatization and secondary traumatic stress disorders. En C. R. Figley (Ed.), Compassion fatigue: Coping with secondary traumatic stress disorder in those who treat the traumatized (Brunner/Mazel Psychological Stress Series No 23; pp. 150-177). Brunner/Mazel.

Pithers, R. T. \& Fogarty, G. J. (1995). Symposium on teacher stress: Occupational stress among vocational teachers. British Journal of Educational Psychology, 65(1), 3-14. https://doi.org/10.1111/j.2044-8279.1995.tb01127.x

Pressley, T. (2021). Factors contributing to teacher burnout during COVID-19. Educational Researcher, 50(5), 325327. https://doi.org/10.3102/0013189X211004138

Qiu, J., Shen, B., Zhao, M., Wang, Z., Xie, B. \& Xu, Y. (2020). A nationwide survey of psychological distress among Chinese people in the COVID-19 epidemic: Implications and policy recommendations. General Psychiatry, 33(2), e100213.

Quan-Haase, A. \& Wellman, B. (2006). Hyperconnected net work: Computer-mediated community in a high-tech organization. En QuanHaase, Anabel \& Wellman, Barry (Eds.), The firm as a collaborative community: Reconstructing trust in the knowledge economy (pp. 281-333). Oxford University Press

$\mathrm{R}$ Development Core Team. (2020). R: A language and environment for statistical computing [Software computacional]. $\mathrm{R}$ Foundation for Statistical Computing. https://www.R-project.org/

Reeves, M. (2012). Efecto del liderazgo directivo en escuelas con altos niveles de vulnerabilidad social. En J. Weinstein \& G. Muñoz (Eds.), ¿Qué sabemos sobre los directores de escuela en Chile? (pp. 307-324). Fundación Chile, Centro de Innovación en Educación \& Pontificia Universidad Católica de Chile, Centro de Estudios de Políticas y Prácticas en Educación.

Reimers, F. M. \& Schleicher, A. (2020). A framework to guide an education response to the COVID-19 pandemic of 2020. Organisation for Economic Co-operation and Development. https://www.aforges.org/wp-content/uploads/2020/04/framework.pdf

Roffey, S. (2012). Pupil wellbeing - Teacher wellbeing: Two sides of the same coin? Educational \& Child Psychology, 29(4), 8-17. https://researchdirect.westernsydney.edu.au/islandora/object/uws:14247

Rogero García, J. (2010). Los tiempos del cuidado. El impacto de la dependencia de los mayores en la vida cotidiana de sus cuidadores. Gobierno de España, Ministerio de Sanidad y Política Social, Instituto de Mayores y Servicios Sociales.

Rubio González, J., Andrade Daigre, P., Fravega Araneda, G., Macalusso Salgado, S. \& Soto Sandoval, A. (2019). Factores psico-socioambientales asociados al estrés laboral en profesores chilenos del ámbito rural y urbano. Propósitos y Representaciones, 7(3), 300311. http://.doi.org/10.20511/pyr2019.v7n3.358

Salanova, M., Martínez, I. M. \& Lorente, L. (2005). ¿Cómo se relacionan los obstáculos y facilitadores organizacionales con el burnout docente?: un estudio longitudinal. Revista de Psicología del Trabajo y las Organizaciones, 21(1-2), 37-54. https://www.redalyc.org/pdf/2313/231317039003.pdf

Saloviita, T. \& Pakarinen, E. (2021). Teacher burnout explained: Teacher-, student-, and organisation-level variables. Teaching and Teacher Education, 97, Article 103221. https://doi.org/10.1016/j.tate.2020.103221

Sánchez Cerón, M. \& Corte Cruz, F. M. S. (2012). La precarización del trabajo. El caso de los maestros de educación básica en América Latina. RLEE: Revista Latinoamericana de Estudios Educativos, 42(1), 25-54. http://www.redalyc.org/pdf/270/27023323003.pdf

Sarwar, F., Panatik, S. A., Sukor, M. S. M. \& Rusbadrol, N. (2021). A job demand-resource model of satisfaction with work-family balance among academic faculty: Mediating roles of psychological capital, work-to-family conflict, and enrichment. SAGE Open, 11(2), Artículo 13. https://doi.org/10.1177/21582440211006142

Schaufeli, W. B. \& Bakker, A. B. (2004). Job demands, job resources, and their relationship with burnout and engagement: A multisample study. Journal of Organizational Behavior, 25(3), 293-315. https://doi.org/10.1002/job.248

Schermelleh-Engel, K., Moosbrugger, H. \& Müller, H. (2003). Evaluating the fit of structural equation models: Tests of significance and descriptive goodness-of-fit measures. Methods of Psychological Research-online, 8(2), 23-74. https://psycnet.apa.org/record/200308119-003

Skaalvik, E. M., \& Skaalvik, S. (2011). Teacher job satisfaction and motivation to leave the teaching profession: Relations with school context, feeling of belonging, and emotional exhaustion. Teaching and Teacher Education, 27(6), 10291038. https://doi.org/10.1016/j.tate.2011.04.001

Skaalvik, E. M. \& Skaalvik, S. (2017). Still motivated to teach? A study of school context variables, stress and job satisfaction among teachers in senior high school. Social Psychology of Education, 20(1), 15-37. https://doi.org/10.1007/s11218-016-9363-9.

Skrondal, A. \& Rabe-Hesketh, S. (2004). Generalized latent variable modeling: Multilevel, longitudinal, and structural equation models. Chapman \& Hall/CRC. https://doi.org/10.1201/9780203489437

Subaldo Suizo, L. (2012). Las repercusiones del desempeño docente en la satisfacción y el desgaste del profesorado [Tesis de doctorado, Universidad de Valencia, España]. RODERIC. Repositori de Contigut Lliure. https://roderic.uv.es/handle/10550/24703

Sutton, R. E. \& Harper, E. (2009). Teachers' emotion regulation. En L. J. Saha \& A. G. Dworkin (Eds.), International handbook of research on teachers and teaching (pp. 389-401). Springer. https://doi.org/10.1007/978-0-387-73317-3_25

Taylor, C., Harrison, J., Haimovitz, K., Oberle, E., Thomson, K., Schonert-Reichl, K. \& Roeser, R. W. (2016). Examining ways that a mindfulness-based intervention reduces stress in public school teachers: A mixed-methods study. Mindfulness, 7(1), 115-129. https://doi.org/10.1007/s12671-016-0620-y

Thoonen, E. E. J., Sleegers, P. J. C., Oort, F. J., Peetsma, T. T. D., \& Geijsel, F. P. (2011). How to improve teaching practices: The role of teacher motivation, organizational factors, and leadership practices. Educational Administration Quarterly, 47(3), 496536. https://doi.org/10.1177/0013161X11400185

Toledo González, M. P. \& Aguilar Pérez, M. (2016). Entre el afecto y las disputas: la casa como espacio laboral feminizado. Espacialidades, 6(1), 192-219. https://www.redalyc.org/pdf/4195/419548242006.pdf

Travers, C. J. \& Cooper, C. L. (1996). Teachers under pressure: Stress in the teaching profession. Routledge.

United Nations Educational, Scientific, and Cultural Organization. (17 de abril de 2020). COVID-19 educational disruption and response. https://en.unesco.org/covid19/educationresponse

Valverde Berrocoso, J. \& Garrido Arroyo, M. C. (2005). La función tutorial en entornos virtuales de aprendizaje: comunicación y comunidad. RELATEC: Revista Latinoamericana de Tecnología Educativa, 4(1), 153-167. https://relatec.unex.es/article/view/195/183

Van Horn, J. E., Taris, T. W., Schaufeli, W. B. \& Schreurs, P. J. G. (2004). The structure of occupational well-being: A study among Dutch teachers. Journal of Occupational and Organizational Psychology, 77(3), 365-375. https://doi.org/10.1348/0963179041752718 
Van Houtven, C. H., DePasquale, N. \& Coe, N. B. (2020). Essential long-term care workers commonly hold second jobs and double- or triple-duty caregiving roles. Journal of the American Geriatrics Society, 68(8), 1657-1660. https://doi.org/10.1111/jgs.16509

Verhavert, S., De Maeyer, S., Donche, V. \& Coertjens, L. (2018). Scale separation reliability: What does it mean in the context of comparative judgment? Applied Psychological Measurement, 42(6), 428-445. https://doi.org/10.1177/0146621617748321

Vernberg, E. M., La Greca, A. M., Silverman, W. K. \& Prinstein, M. J. (1996). Prediction of posttraumatic stress symptoms in children after hurricane Andrew. Journal of Abnormal Psychology, 105(2), 237-248. https://doi.org/10.1037/0021-843x.105.2.237

Wang, Q. \& Huang, C. (2018). Pedagogical, social and technical designs of a blended synchronous learning environment. British Journal of Educational Technology, 49(3), 451-462. https://doi.org/10.1111/bjet.12558

Weathers, J. M. (2011). Teacher community in urban elementary schools: The role of leadership and bureaucratic accountability. Education Policy Analysis Archives, 19, Artículo 3. https://doi.org/10.14507/epaa.v19n3.2011

Xanthopoulou, D., Bakker, A. B., Demerouti, E., \& Schaufeli, W. B. (2007). The role of personal resources in the job demands-resources model. International Journal of Stress Management, 14(2), 121-141. https://doi.org/10.1037/1072-5245.14.2.121

Xie, Z. \& Yang, J. (15 de marzo de 2020). Autonomous learning of elementary students at home during the COVID-19 epidemic: A case study of the second elementary school in Daxie, Ningbo, Zhejiang Province, China. SSRN. https://doi.org/10.2139/ssrn.3555537

Yao, J., Rao, J., Jiang, T. \& Xiong, C. (2020). What role should teachers play in online teaching during the COVID-19 pandemic? Evidence from China. Science Insights Education Frontiers, 5(2), 517-524. https://doi.org/10.15354/sief.20.ar035

Yin, H. -b. \& Lee, J. C. -K. (2012). Be passionate, but be rational as well: Emotional rules for Chinese teachers' work. Teaching and Teacher Education, 28(1), 56-65. https://doi.org/10.1016/j.tate.2011.08.005

$\mathrm{Yu}$, C. -Y. (2002). Evaluating cutoff criteria of model fit indices for latent variable models with binary and continuous outcomes (Publicación $N^{\circ}$ 3066425). [Disertación de doctorado, University of California]. ProQuest Dissertations \& Theses Global.

Zavala Zavala, J. (2008). Estrés y burnout docente: conceptos, causas y efectos. Educación (Pontificia Universidad Católica del Perú), 17(32), 67-86. https://revistas.pucp.edu.pe/index.php/educacion/article/view/1802 


\title{
ANEXO
}

\section{INSTRUMENTO UTILIZADO}

El instrumento original tenía 34 páginas e incluía 113 ítemes. En este artículo usamos un subconjunto de estas preguntas. Este anexo resume de forma editada las preguntas que fueron incluidas en este estudio, de manera de aumentar la claridad. En su versión original algunas de estas preguntas eran presentadas en conjunto con otros reactivos que no fueron utilizados por parte del estudio.

\section{Preguntas de caracterización sociodemográfica}

\subsection{Género}

\section{Masculino \\ Femenino \\ Prefiero no decir}

$1.2 \mathrm{Edad}$

1.3. ¿En qué zona se ubica su establecimiento?

Urbana

1.4. Dependencia administrativa del establecimiento

\author{
JUNJI \\ Integra \\ Vía Transferencia de Fondos (VTF) \\ Municipal o Servicio Local de Educación \\ Particular Subvencionado \\ Particular Pagado \\ Administración Delegada
}

1.5. Tamaño del establecimiento

1 a 500 estudiantes

501 a 1.000 estudiantes

Más de 1.000 estudiantes

1.6. ¿Cuántos años de experiencia tiene en el sistema educativo?

1.7. ¿Vive con personas menores de 18 años que dependan de usted? (Hijo, nieto, sobrino, etc.) Seleccione todas las opciones que correspondan

No

Sí, al menos uno de ellos tiene menos de 1 año

Sí, al menos uno de ellos tiene entre 1 y 4 años

Sí, al menos uno de ellos tiene entre 5 y 6 años

Sí, al menos uno de ellos tiene entre 7 y 14 años

Sí, al menos uno de ellos tiene entre 15 y 18 años

1.8. ¿Es usted responsable del cuidado de algún familiar o conocido mayor de edad al interior del hogar?

No

Sí, colaboro en el cuidado

Sí, soy el cuidador principal

1.9. Pensando en el periodo de cierre de su establecimiento, ¿de qué manera está haciendo clases? Seleccionar a la que dedica más tiempo

En vivo (de manera virtual) sin posibilidad de interacción con estudiantes

En vivo (de manera virtual) con posibilidad de interacción con estudiantes

Grabadas (video o audio), enviadas o subidas a alguna plataforma

No estoy haciendo clases, pero hago entrega de guías y recursos pedagógicos (online o físicamente)

No estoy haciendo clases 
1.10. Tamaño promedio de el/los curso/s donde imparte clases

Menos de 15 estudiantes

Entre 15 y 30 estudiantes

Más de 30 estudiantes

1.11. ¿En qué nivel o cursos realiza clases? (Si es más de uno, seleccione el que concentre mayor número de horas)

Sala cuna

Niveles medios

Niveles de transición

1ro a 4to básico

5to a 8 vo básico

Educación media Humanista Científico

Educación media Técnico Profesional (general)

Educación media Técnico Profesional o Artística

\section{Angustia psicológica}

2.1. Desde el cierre del establecimiento, en una escala de 1 a 5 , donde 1 es "nada" y 5 es "muy", ¿qué tanto se ha sentido Ansioso?

2.2. Desde el cierre del establecimiento, en una escala de 1 a 5 , donde 1 es "nada" y 5 es "muy", ¿qué tanto se ha sentido Enojado?

2.3. Desde el cierre del establecimiento, en una escala de 1 a 5 , donde 1 es "nada" y 5 es "muy", ¿qué tanto se ha sentido Relajado?

2.4. Desde el cierre del establecimiento, en una escala de 1 a 5 , donde 1 es "nada" y 5 es "muy", ¿qué tanto se ha sentido Cansado?

2.5. Desde el cierre del establecimiento, en una escala de 1 a 5, donde 1 es "nada" y 5 es "muy", ¿qué tanto se ha sentido Preocupado?

2.6. Desde el cierre del establecimiento, en una escala de 1 a 5 , donde 1 es "nada" y 5 es "muy", ¿qué tanto se ha sentido Abrumado?

2.7. Pensando en el nivel de estrés desde el cierre del establecimiento, en una escala de 1 a 5 , donde 1 es "nada estresado" y 5 es "muy estresado", ¿en qué nivel se encuentra usted?

\section{Recursos para el trabajo a distancia}

3.1. Considerando la situación actual, en una escala de 1 a 5 , donde 1 es "no tengo" y 5 es "tengo y es óptimo", ¿cómo evalúa el Espacio de trabajo para desarrollar su trabajo desde el hogar?

3.2. Considerando la situación actual, en una escala de 1 a 5 , donde 1 es "no tengo" y 5 es "tengo y es óptimo", ¿cómo evalúa su Señal de teléfono para desarrollar su trabajo desde el hogar?

3.3. Considerando la situación actual, en una escala de 1 a 5 , donde 1 es "no tengo" y 5 es "tengo y es óptimo", ¿cómo evalúa su Conexión a internet en el hogar el para desarrollar su trabajo desde el hogar?

3.4. Considerando la situación actual, en una escala de 1 a 5 , donde 1 es "no tengo" y 5 es "tengo y es óptimo", ¿cómo evalúa su computador personal el para desarrollar su trabajo desde el hogar?

\section{Apoyo directivo}

4.1. En comparación a la manera en que trabajaba antes del cierre del establecimiento, en una escala de 1 a 5 , donde 1 es "menos que antes" y 5 es "más que antes", actualmente ¿cómo evaluaría las siguientes afirmaciones? Me siento apoyado por el equipo directivo en el ámbito pedagógico

4.2. En comparación a la manera en que trabajaba antes del cierre del establecimiento, en una escala de 1 a 5 , donde 1 es "menos que antes" y 5 es "más que antes", actualmente icómo evaluaría las siguientes afirmaciones? Me siento apoyado por el equipo directivo en el ámbito emocional

4.3. Considerando la situación actual, en una escala de 1 a 5 , donde 1 es "no tengo" y 5 es "tengo y es óptimo", ¿cómo evalúa las siguientes instancias o elementos para desarrollar su trabajo desde el hogar? Orientación técnico-pedagógica desde el establecimiento 


\section{Trabajo colaborativo}

5.1. ¿En promedio, desde el cierre del establecimiento, cuántas veces se ha comunicado por motivos laborales con las siguientes personas? Colegas (Equipo directivo o técnico pedagógico)

5.2. En base a esa comunicación, ¿qué tan satisfecho se ha sentido? Colegas (Equipo directivo o técnico pedagógico)

5.3. En comparación a la manera en que trabajaba antes del cierre del establecimiento, en una escala de 1 a 5 , donde 1 es "menos que antes" y 5 es "más que antes", actualmente ¿cómo evaluaría las siguientes afirmaciones? Trabajo colaborativamente con mis colegas

\section{Tiempo disponible}

6.1. Considerando la situación actual, en una escala de 1 a 5 , donde 1 es "no tengo" y 5 es "tengo y es óptimo", ¿cómo evalúa el Tiempo para preparar clases y/o material para desarrollar su trabajo desde el hogar?

6.2. En comparación a la manera en que trabajaba antes del cierre del establecimiento, en una escala de 1 a 5 , donde 1 es "menos que antes" y 5 es "más que antes", actualmente ¿cómo evaluaría las siguientes afirmaciones? Puedo compatibilizar bien los tiempos de trabajo doméstico y trabajo pedagógico

Fecha de recepción: Agosto de 2020.

Fecha de aceptación: Agosto de 2021. 